\title{
Optimal finite locally resonant metafoundations enhanced with nonlinear negative stiffness elements for seismic protection of large storage tanks
}

\author{
Moritz Wenzel $^{\mathrm{a}, *}$, Oreste S. Bursi ${ }^{\mathrm{a}}$, Ioannis Antoniadis ${ }^{\mathrm{b}}$ \\ ${ }^{a}$ University of Trento, Department of Civil, Environmental and Mechanical Engineering \\ ${ }^{b}$ National Technical University of Athens, School of Mechanical Engineering
}

\begin{abstract}
Metamaterials represent a new trend in the field of seismic engineering. Their capacity to attenuate waves at the superstructure level is highly desirable and sought after in recent years. One of their main drawbacks to date, is the excessive size of the necessary resonators and, consequently, the uneconomic design they require. In order to tackle this problem, we apply the concept of negative stiffness to a metamaterial-based foundation system and analyse the potential improvements such a mechanism may have on the metamaterial as well as the coupled structural behaviour. Since negative stiffness is a property that cannot be achieved through conventional measures, a novel mechanism, designed for the implementation in periodic metamaterial-based structures, is proposed herein. The inevitable nonlinearity of the mechanism will be discussed and taken into account, while the advantages of the negative stiffness element (NSE) will be treated analytically and verified numerically. Additionally, through an optimization in the frequency domain and nonlinear time history analyses (THAs), the performance of the system coupled with a fuel storage tank is elaborated. With only $50 \%$ of the theoretically allowable NSE value, the foundation system could be reduced to $1 / 3$ of its size. Furthermore, the nonlinear effect of the device has proven to diminish the band gap of the periodic system, which led us to introduce nonlinearity parameters that can help avoid the strongly nonlinear
\end{abstract}

\footnotetext{
${ }^{*}$ Corresponding author
} 
range. In sum, this article tackles three problems that are intertwined: (i) reducing the size of metamaterial-based structures; (ii) the design of a mechanism that exerts a negative stiffness in a periodic structure; and (iii) the study of the inevitable nonlinearity of NSEs and the subsequent effect on the metamaterial behaviour.

Keywords: Metafoundation, Negative Stiffness, Seismic protection, Fuel Storage Tanks, Nonlinear Metamaterials

\section{Introduction}

Metamaterials are entering the field of seismic engineering and other research areas with a variety of interesting structures. The two most prevalent concepts in the field of seismic protection are phononic crystals [1] and locally

5 resonant metamaterials [2], where both are able to create the so called bad gap phenomenon. Band gaps signify frequency regions where waves cannot propagate through the material and are therefore able to provide new solutions to existing vibration problems. For the present work we focus on locally resonant materials, due to their ability to attenuate waves at wave lengths much greater than their unit cell size, which is a particularly important property for seismic metamaterials. To date, locally resonant materials have been used to conceive foundation systems [3, 4, 5, 6, 7] and wave barriers [8, 9, 10, 11. While metabarriers have the advantage of being placed besides the structure, and can therefore be installed after the completion of the building, they can only attenuate surface waves. Metamaterial-based foundations on the other hand, can in principle attenuate any type of incoming wave, but have to be placed below the structure of interest, hence limiting their application to new buildings. The present work is concerned with foundation systems, which show a variety of different designs and applications in the current literature. A particularly interesting foundation was proposed by Cheng and Shi [5] who conceived a system tuned to the ground motion for the protection of nuclear power plants. Their foundation showed different band gaps for the vertical and horizontal direction, thereby ad- 
dressing the vertical component of earthquakes. This is especially relevant for high consequence structures like nuclear power plants, since classical isolation systems, like concave sliding bearings, are not able to address the vertical motion [12. Besides this, also Casablanca et al. [7] developed an interesting foundation based on concrete plates separated by Teflon sliding surfaces and verified its behaviour with laboratory experiments. Their experiments clearly depicted that these types of structures are feasible with common construction materials and can exert the band gap phenomenon. However, neither Cheng and Shi nor Casablanca et al. took the feedback from the structure into account. La Salandra et al. [4] on the other hand designed a foundation system and conducted a study on the most influencing factors on the attenuation behaviour. Two important findings shall be mentioned, namely, the influence of the stiffness and the non-negligibility of the feedback of the superstructure. Subsequently, Basone et al. 13] developed a foundation system based on their results and conceived an optimization procedure that can take a structure as well as an ensemble of expected ground motions into account. However, their design shows significant restrictions in terms of effectiveness due to the constraints given by the governing building codes (i.e. Eurocode 3 and 8, [14, 15]). Besides this, an experimental study on the coupling effects between a tank isolated with a metamaterial-based foundation and a pipeline suggested that this type of foundation may provide a compromise between base shear attenuation and horizontal displacement [16], which is a property that cannot be obtained with classical isolation systems. 45 Further worth mentioning is the work of Witarto et al. [17 who studied the application of metamaterial-based systems to small scale nuclear reactors; and the work done by Ungureanu et al. [18] who used auxectic like materials to protect high-rise buildings. Finally, a comprehensive review of seismic metamaterials including metabarriers as well as foundation systems was given recently 50 by $\mathrm{Mu}$ et al. [19. From their review one can clearly conclude that one of the most pressing problems of metamaterial-based foundations is the excessive size necessary to obtain a functional foundation. However, two advantages may become attainable through such foundations in future, namely: (i) attenuation of 
the vertical component [5] and rocking motions [20], which cannot be addressed by traditional base isolation systems [21]; and (ii) a compromise between base shear reduction and horizontal displacement [16]. One idea to improve the performance of a metamaterial-based system was proposed by Antoniadis et al. [22] who showed that a negative stiffness element (NSE) inserted in the resonator mechanism could potentially improve the system behaviour significantly.

60 Note that this is not an effective negative stiffness as discussed in e.g. [23, but a composite spring system where the resulting force assists motion and does not oppose it. Note that Antoniadis et al. [22] included only a conceptual negative stiffness element that would exert the desirable amplification force, while a design for an actual mechanism that could be applied to a periodic structure was still missing. To date, most proposals including negative stiffness and metamaterials aim at the continuum level 24, 25, while Morris et al. 26] conducted an experimental study on such a continuous metamaterial with buckling type instabilities and showed the energy dissipation capabilities of the structured medium. These proposals are interested mainly in the material level, and therefore, do not investigate the application to a structure or the inevitable nonlinear effect of an NSE on the band gap.

It is worth noting that research work on nonlinear metamaterials is still limited and primarily concerned with weakly nonlinear resonant chains. A perturbation approach for the dispersion analysis of weakly nonlinear chains has 75 been proposed by Chakraborty and Mallik [27, which clearly depicts that: (i) solutions to nonlinear wave equations are amplitude dependent; (ii) wave amplitudes influence their own propagation characteristics, the so-called self-action; and (iii) analysis methods in the presence of self-action often do not trace all solutions when more than one dominant component is involved. Another neat 80 approach to calculating the band gaps for such materials relies on the harmonic balance method (HBM) as has been demonstrated by Lazarov and Jensen [28]. Banerjee et al. 29] on the other hand provide a comprehensive review of 1D metamaterials including materials with nonlinear oscillators. Both showed classical bi-atomic lattices with nonlinear oscillators, e.g. Duffing oscillator, pendu- 
lum, impacting resonators, and concluded that an increase in elastic nonlinearity, entails a shift and an elongation of the band gap. Based on the current state of the art, the present work conceives a new mechanism applicable to periodic structures, which is able to reduce the size of metamaterial-based foundations. In order to present a realistic application, a fuel storage tank was chosen as a su-

90 perstructure and its feedback taken into account when designing and optimizing the foundation. Note that fuel storage tanks represent the most vulnerable and consequence intensive components of industrial plants during earthquakes, and that their seismic protection is still an ongoing issue [30, 31, 32, 33, 34. The coupled Metafoundation tank system is analysed on its performance for various foundation heights and different levels of applied negative stiffness herein. Note that the practical application includes only a one layered foundation, while further analyses, carried out on the system considered as a periodic structure, shed light on the wave propagation in nonlinear negative stiffness enhanced materials.

\subsection{Scope}

The present work tackles three main research issues, namely: (i) Size reduction of metamaterial-based structures for seismic applications; (ii) development of an NSE that can be implemented in a metamaterial; and (iii) investigation of the inevitable nonlinear behaviour. The manuscript discusses these issues in the following order: Section 2 elaborates the structure, the foundation, and the mechanism and shows the simplified dynamic system used in the subsequent analyses; Section 3 shows the metamaterial-like behaviour of a periodic system with and without considering the nonlinear effect; Section 4 demonstrates an optimization algorithm for the optimal design of the foundation; Section 5 investigates the behaviour of the complete coupled and optimized structure under real seismic action; and Section 6 closes the paper with conclusions and future developments. 


\section{Description of the structure}

The Metafoundation was initially conceived in [4] and later developed and designed according to common construction standards by [13]. The proposed foundation is based on steel columns that support concrete slabs, with resonators placed in between the columns, in order to provide the system with its locally resonant properties, see Figure 1(a). Additionally, for an improved performance of the system, a new type of NSE is designed and implemented in the structure by mounting it to the columns and resonators as displayed in Figure 11(b) and 2. It is further worth noting that the columns govern the horizontal stiffness of the system, which has a significant impact on the functionality of the foundation during earthquakes as shown by [4, 13]. They also investigated multiple foundation set-ups, where the one layered design turned out to be the most material efficient version, due to construction standard requirements on the steel columns. Therefore, the present work treats a one layered foundation for feasibility investigations, while a multi-layered foundation system will be discussed only on its wave propagation behaviour. Along these lines, 3 different foundations, namely the FULL, REDUCED and MINIMAL systems, are studied herein, which are distinguished by their heights and column cross sections, as listed in Table 1. Note that Figure 1 shows the MINIMAL foundation layout where the foundation height amounts to $1 \mathrm{~m}$, while the column width is 0.17 m. Moreover, the columns consist of steel hollow sections with a plate thickness of $0.03 \mathrm{~m}$ for all foundation set-ups. It is worth mentioning that each systems has been designed to remain elastic for a return period of 2475 years at the site Priolo Gargallo, Italy, according to Eurocode 3 and 8 [14, 15]. Finally, due to the double symmetry of the structure, the system is condensed to a $1 \mathrm{D}$ representation that propagates only shear type waves to the superstructure, which is elaborated in Sections 2.1 and 2.2

\subsection{Negative stiffness element NSE}

Since a simple spring with a negative effective stiffness does not exist, it is necessary to design a suitable mechanism that can exert the desired forces. For 

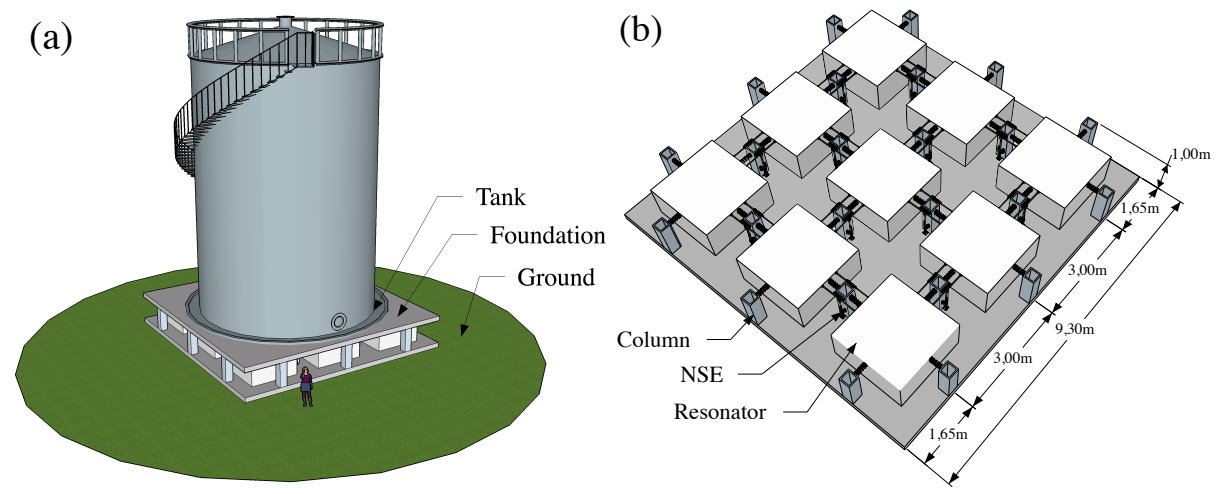

Figure 1: Layout of the Metafoundation for the MINIMAL system: (a) Isometric view; (b) Internal view of the foundation components.

Table 1: Geometric properties of the various foundation setups.

\begin{tabular}{lccc}
\hline & FULL & REDUCED & MINIMAL \\
\hline Foundation height & $3 \mathrm{~m}$ & $2 \mathrm{~m}$ & $1 \mathrm{~m}$ \\
Resonator height & $2.7 \mathrm{~m}$ & $1.7 \mathrm{~m}$ & $0.75 \mathrm{~m}$ \\
Column width & $0.3 \mathrm{~m}$ & $0.24 \mathrm{~m}$ & $0.17 \mathrm{~m}$ \\
Comp. mem. length $l$ & $2.7 \mathrm{~m}$ & $1.7 \mathrm{~m}$ & $0.7 \mathrm{~m}$ \\
\hline
\end{tabular}


the mechanism at hand we employ a compression member and subject it to a prestress force, as depicted in Figure 2 Note that the compression member is guided vertically along the column and horizontally along the slab above, in order to allow for an inclination in the displaced state. Due to this inclination, the compression member releases the stored potential energy from the prestressed spring as a horizontal force pair on the resonators and the columns, see Figure 3. Furthermore, as can be seen from Figure 1, the mechanism is placed between resonators on both sides of the relevant columns, which amounts to a total of 12 mechanisms per horizontal axis of the foundation. The 9 resonators on the other hand are assumed to slide on frictionless surfaces and have identical properties.

\subsubsection{NSE analytical model}

All resonators, columns and installed mechanisms are identical, and are therefore condensed to one resonator, one column and one mechanism for one layer of foundation. The kinematics of the system can subsequently be simplified as depicted in Figure 3(a), where $k_{1}$ denotes the condensed stiffness of all columns in one layer, $m_{2}$ is the mass of all resonators, $m_{1}$ represents the mass of the concrete slab, $k_{R}$ denotes the condensed spring stiffness of all springs that support the resonators (see also Figure 2), and $k_{p}$ is the stiffness of all prestressed springs. In order to substitute the mechanism with an equivalent nonlinear spring, the force equilibrium needs to be formulated on the displaced system, as shown in Figure 3(b). Here, $F_{R}$ denotes the horizontal force applied to the resonator and the bottom slab; $F_{S}$ describes the vertical force applied by the prestressed spring; $l$ is the length of the compression member; $u$ is the vertical displacement of the member; and $v$ is the horizontal displacement of the resonator and the compression member at its top. When establishing the force equilibrium around the member as depicted in Figure 3 (b), the following 


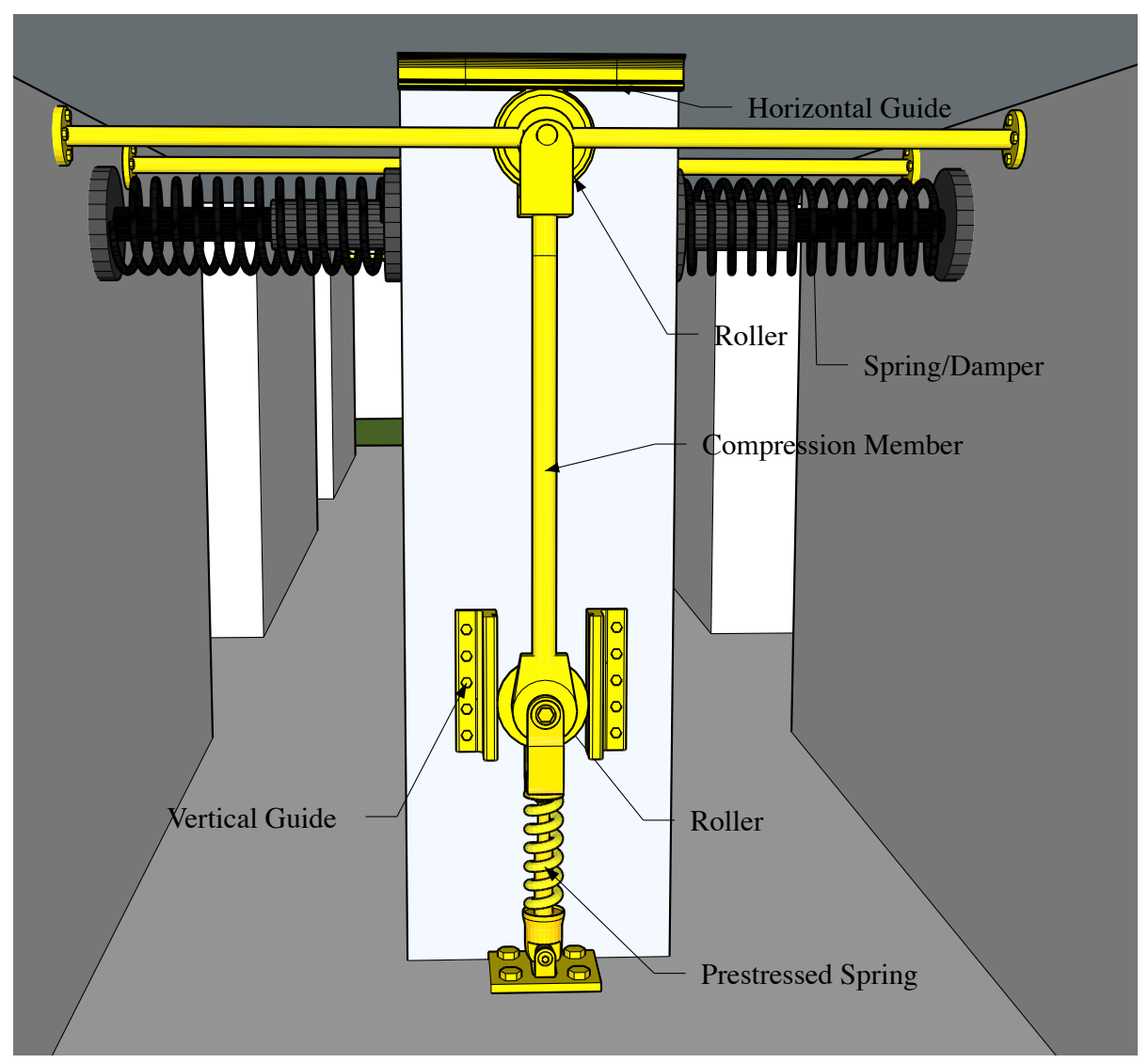

Figure 2: Negative stiffness mechanism.
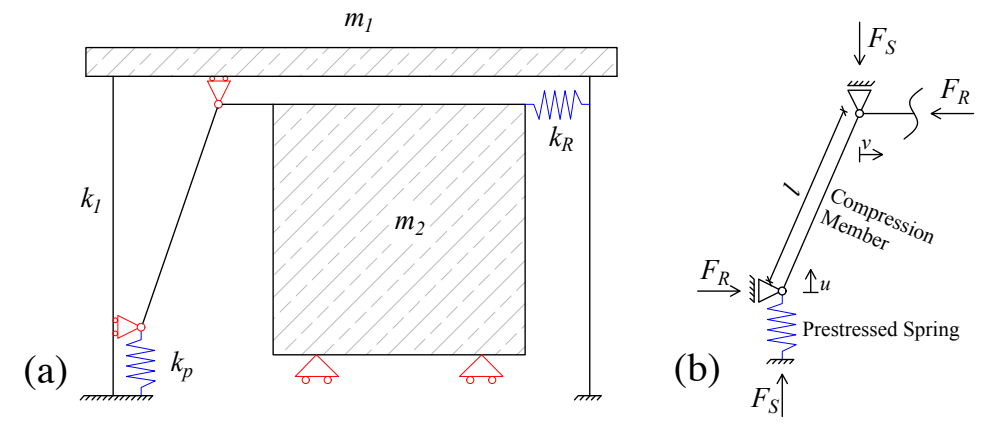

Figure 3: Kinematic system in the displaced state: (a) Resonator and mechanism in displaced state; (b) Force equilibrium on the displaced system. 
geometrical relationships can be drawn,

$$
\begin{aligned}
F_{S} & =u k_{p}-P \\
F_{R} & =\frac{F_{S}}{(l-u) / l} \frac{v}{l} \\
(l-u)^{2} & =l^{2}-v^{2}
\end{aligned}
$$

Here, $P$ is the prestress force applied to the spring when the member is in its vertical position. After some algebra the horizontal force $F_{R}$ can be put in relation to the displacement of the resonator $v$ with,

$$
F_{R}(v)=\frac{v\left(-P+k_{p}\left(l-\sqrt{l^{2}-v^{2}}\right)\right)}{\sqrt{l^{2}-v^{2}}}
$$

Eq. (4) clearly is a nonlinear relation for the force displacement path of the resonator, due to the relationship between the displacements $u$ and $v$. It is important to note that the denominator of this function becomes 0 for $v \rightarrow l$, which has the effect of an infinite stiffness at $v=l$. The length of the mechanism, therefore, plays a significant role in the behaviour of the nonlinearity. Furthermore, a Taylor series approximation of (4) at the origin is also desired, in order to allow simplified nonlinear calculations in the frequency domain with the HBM. With the classical formulation of the Taylor series,

$$
T(x)=\sum_{n=0}^{\infty} \frac{f^{n}(a)}{n !}(x-a)^{n}
$$

the force-displacement relationship (4) can be rewritten for a 3rd order approximation at the origin, with $a=0$, and $n \in\{0,1,2,3\}$, as,

$$
F_{R}(v)=-\frac{P}{l} v+\frac{k_{p} l-P}{2 l^{3}} v^{3}+H O\left(v^{5}\right)
$$

The behaviour of the mechanism can now be dissected into a negative linear and positive nonlinear part,

$$
F_{R}(v)=a_{N S E} v+b_{N S E} v^{3}
$$

with,

$$
a_{N S E}=-\frac{P}{l}=k_{N}
$$




$$
b_{N S E}=\frac{k_{p} l-P}{2 l^{3}}=\frac{k_{p}}{2 l^{2}}\left(1-\frac{P}{k_{p} l}\right)
$$

From these expressions the maximal stiffness of the mechanism appears at the initial configuration and amounts to $k_{N}=a_{N S E}=-P / l$, which will be used as an approximation of the NSE for linear analyses. Furthermore, since $k_{p}$ appears exclusively in the nonlinear part of the polynomial approximation, it can be used to tune the nonlinear shape of the mechanisms force displacement path. Note that when $k_{p} l$ results in a value smaller than $P$, the system is subjected to softening instead of hardening, which is physically not meaningful for the system under study. The limits of the mechanism can therefore be set to $\frac{P}{l} \leq k_{p} \leq \infty$ and a dimensionless nonlinearity parameter established with,

$$
\epsilon=1-\frac{P}{k_{p} l}, \quad 0 \leq \epsilon \leq 1
$$

When $\epsilon \rightarrow 0$ the system behaves linear, while when $\epsilon \rightarrow 1$ the nonlinear component becomes infinite and the system enters it's nonlinear state immediately. The following values were chosen for the sake of demonstration for Figure 4(a), $l=2.7 \mathrm{~m}, P=10000 \mathrm{kN}$ and $\epsilon$ as $0,0.5,0.75$, and 0.9 .

Besides this, a value of $\epsilon=0.95$ was regarded as a realistic value for all set-ups, as it resulted in prestress distances of the prestressed spring equal to $13.5 \mathrm{~cm}, 8.5 \mathrm{~cm}$, and $3.5 \mathrm{~cm}$ for the FULL, REDUCED, and MINIMAL system, respectively. Since the length of the compression member plays a vital role in the nonlinear behaviour, a second force-displacement diagram was investigated with length $l$ of $1,0.5$ and $0.2 \mathrm{~m}$ for $\epsilon=0.95$, and displayed in Figure 4(b). Clearly, the linear approximation is very close to the exact path for small displacements, which is desirable not only for the sake of simplicity, but also for the later proposed optimization algorithm, based on computations in the frequency domain.

\subsection{Dynamic system}

Once the mechanism can be substituted with a simple nonlinear spring endowed with the force-displacement relationship of Eq. (4) or (7), the dynamic 

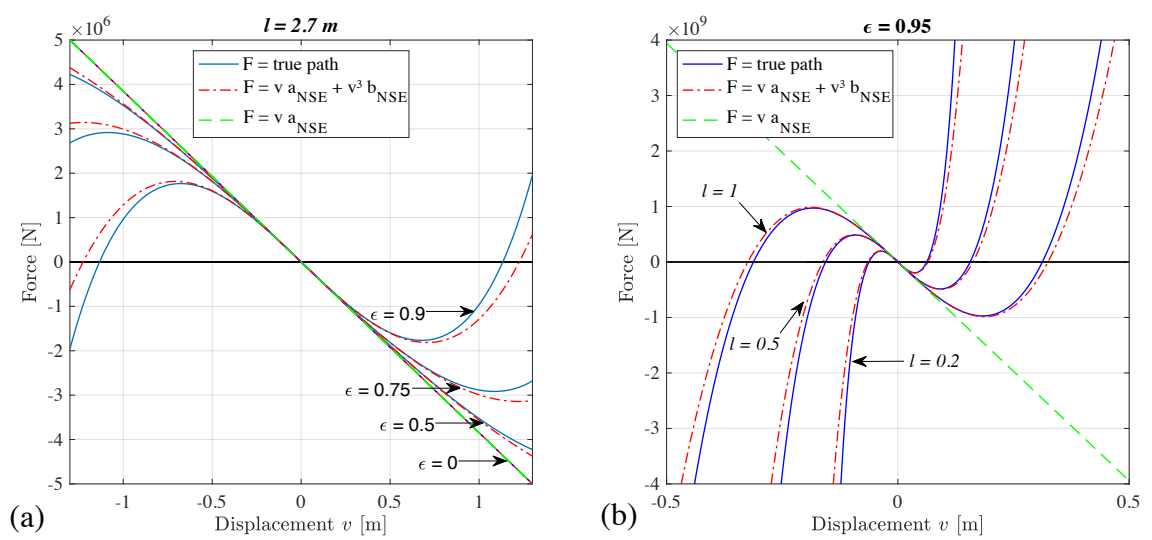

Figure 4: Force displacement path: (a) Variation of parameter $\epsilon$; (b) Variation of member length $l$.

system can be established for the condensed structure as depicted in Figure 5(a). Here, a fuel storage tank with a diameter of $8 \mathrm{~m}$, a height of $12 \mathrm{~m}$ and a steel wall thickness of $6 \mathrm{~mm}$ is used as a superstructure and modelled as a 2 degree of freedom (DOF) system. The two DOFs represent the impulsive and convective mode according to the procedure proposed by Malhotra et al. 35] and are characterized by their stiffness $k_{i}, k_{c}$ and masses $m_{i}, m_{c}$, respectively. In the interest of brevity, the procedure is not elaborated here, while only the stiffness, mass and damping coefficients are listed in Table 2. The reader may note that in Figure 5 the stiffness of the resonators $k_{R}$ is a compound stiffness comprised of $k_{F}-k_{N}$. Here, $k_{N}$ represents the linearized stiffness of the NSE, which also corresponds to the maximal negative stiffness value, while $k_{F}$ denotes that part of $k_{R}$ that determines the resonant frequency of the resonator. Modelling the springs in this way has the following advantages: (i) when $k_{N}$ is small, $k_{F}$ becomes the dominant stiffness and can be used to evaluate the eigenfrequency of the resonator with $\omega_{R}=\sqrt{k_{F} / m_{2}}$; and (ii) if $k_{F}$ becomes small, the local stability of the resonator is still fulfilled by the opposing positive stiffness $-k_{N}$. Furthermore, in Figure 5 the displacement of the foundations top slab and resonator are denoted with $u_{1}$ and $u_{2}$, respectively, while the relative 
Table 2: Parameters for the discretized system.

\begin{tabular}{lrrr}
\hline Parameter & FULL & REDUCED & MINIMAL \\
\hline$m_{1}[\mathrm{~kg}]$ & $5.88358 \mathrm{e}+04$ & $5.00074 \mathrm{e}+04$ & $4.36692 \mathrm{e}+04$ \\
$m_{2}[\mathrm{~kg}]$ & $2.67907 \mathrm{e}+05$ & $1.78605 \mathrm{e}+05$ & $7.93800 \mathrm{e}+04$ \\
$k_{1}[\mathrm{~N} / \mathrm{m}]$ & $8.50176 \mathrm{e}+08$ & $1.36080 \mathrm{e}+09$ & $3.30624 \mathrm{e}+09$ \\
$k_{F}[\mathrm{~N} / \mathrm{m}]$ & to be evaluated & to be evaluated & to be evaluated \\
$k_{N}[\mathrm{~N} / \mathrm{m}]$ & to be evaluated & to be evaluated & to be evaluated \\
$c_{R}[\mathrm{Ns} / \mathrm{m}]$ & to be evaluated & to be evaluated & to be evaluated \\
$m_{i}[\mathrm{~kg}]$ & $4.51666 \mathrm{e}+05$ & $4.51666 \mathrm{e}+05$ & $4.51666 \mathrm{e}+05$ \\
$m_{c}[\mathrm{~kg}]$ & $8.57730 \mathrm{e}+04$ & $8.57730 \mathrm{e}+04$ & $8.57730 \mathrm{e}+04$ \\
$k_{i}[\mathrm{~N} / \mathrm{m}]$ & $8.35184 \mathrm{e}+08$ & $8.35184 \mathrm{e}+08$ & $8.35184 \mathrm{e}+08$ \\
$k_{c}[\mathrm{~N} / \mathrm{m}]$ & $3.86480 \mathrm{e}+05$ & $3.86479 \mathrm{e}+05$ & $3.86479 \mathrm{e}+05$ \\
$c_{i}[\mathrm{Ns} / \mathrm{m}]$ & $1.94223 \mathrm{e}+06$ & $1.94222 \mathrm{e}+06$ & $1.94222 \mathrm{e}+06$ \\
$c_{c}[\mathrm{Ns} / \mathrm{m}]$ & $1.82070 \mathrm{e}+03$ & $1.82070 \mathrm{e}+03$ & $1.82070 \mathrm{e}+03$ \\
\hline
\end{tabular}

ground displacement is represented with $u_{g}$.

For the damping of the structure, we chose a Rayleigh model with 5\% damping at 0.5 and $7 \mathrm{~Hz}$ applied to the structure without resonators and tank. The resonators on the other hand are assigned with a linear visco-elastic damper, denoted with $c_{R}$, which works in parallel with $k_{R}$, and is also subjected to the optimization procedure described later in this work. Furthermore, the two DOFs of the tank have been endowed with damping values of $5 \%$ and $0.5 \%$ for the impulsive and convective mode, respectively, and are denoted with $c_{i}$ and $c_{c}$. All relevant values of the condensed dynamic system for all subsequent analyses are shown in Table 2

Besides this, the proposed foundation will be analysed not only as a one layered system for the protection of fuel storage tanks, but also on its wave propagation properties when arranged as a periodic stack. For this purpose, a multi-layered foundation can be imagined as depicted in Figure 5(b), where one layer represents the unit cell of the system. 
(a)

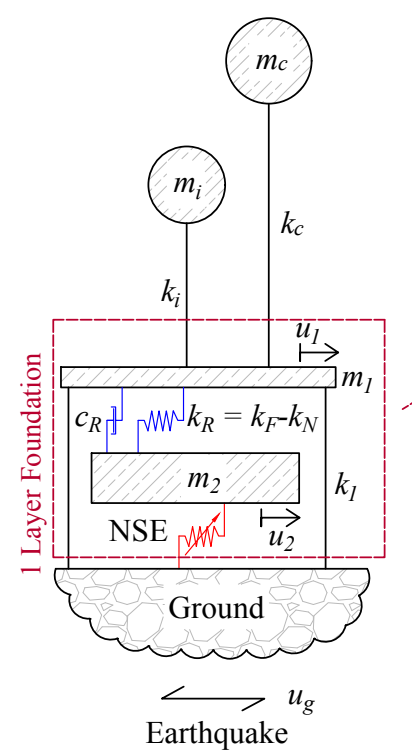

(b)

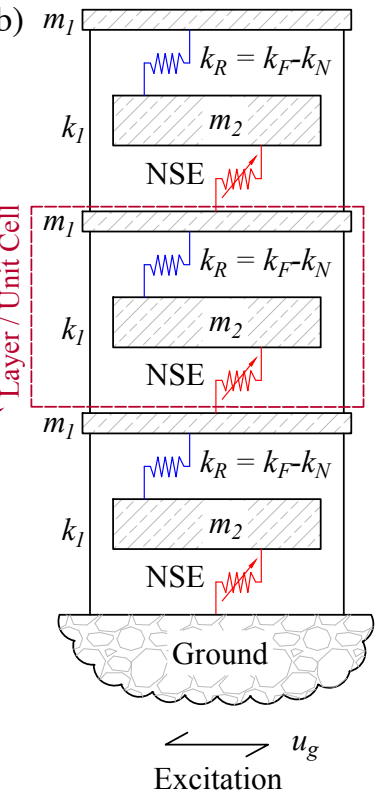

Figure 5: Dynamic systems: (a) Coupled foundation-tank system; (b) Foundation modelled as a periodic structure.

205

\subsection{Stability condition of the system}

Due to the use of a local instability, it is necessary to determine the maximal allowable parameters, where the local instability does not impose a global instability on the system. The failure mode interesting for this analysis is the collapse of the unit cell due to an excessive negative force in the NSE, which leads to the analysis of a single layer without tank, see also Figure 5(a). The system of equations of motion (EOMs) can be written as,

$$
\begin{aligned}
& m_{1} \ddot{u}_{1}(t)+k_{1} u_{1}(t)+\left(k_{F}-k_{N}\right) u_{1}(t)-\left(k_{F}-k_{N}\right) u_{2}(t)=m_{1} \ddot{u}_{g}(t) \\
& m_{2} \ddot{u}_{2}(t)-\left(k_{F}-k_{N}\right) u_{1}(t)+\left(k_{F}-k_{N}\right) u_{2}(t)+k_{N} u_{2}(t)=m_{2} \ddot{u}_{g}(t)
\end{aligned}
$$

Here, $u_{1}$ denotes the displacement of the slab, $u_{2}$ describes the motion of the resonator, and $\ddot{u}_{g}$ is the relative ground acceleration. Under harmonic excitation $\ddot{u}_{g}(t)=u_{g 0} e^{i \omega t}$ the displacement responses can be assumed harmonic with $u_{1}(t)=u_{10} e^{i \omega t}$, and $u_{2}(t)=u_{20} e^{i \omega t}$, and the system expressed in the frequency 
domain (after dividing by $e^{i \omega t}$ on both sides) with,

$$
\begin{array}{r}
\left(-\omega^{2}+k_{1}+k_{F}-k_{N}\right) u_{10}-\left(k_{F}-k_{N}\right) u_{20}=m_{1} u_{g 0} \\
\left(-\omega^{2} m_{2}+k_{F}-k_{N}+k_{N}\right) u_{20}-\left(k_{F}-k_{N}\right) u_{10}=m_{2} u_{g 0}
\end{array}
$$

When substituting (14) into (13) and rearranging the terms, the frequency response function FRF for $u_{10}$ can be written as,

$$
u_{10}=\frac{k_{N} m_{2}-k_{F} m_{1}-k_{F} m_{2}+m_{1} m_{2} \omega^{2}}{k_{N}^{2}-k_{1} k_{F}-k_{F} k_{N}+\left(k_{F} m_{1}+k_{1} m_{2}+k_{F} m_{2}-k_{N} m_{2}\right) \omega^{2}-m_{1} m_{2} \omega^{4}} u_{g 0}
$$

The denominator of the FRF, being the characteristic equation of the system, takes up the form,

$$
y=\omega^{4} \alpha_{1}+\omega^{2} \alpha_{2}+\alpha_{3}
$$

with,

$$
\begin{aligned}
& \alpha_{1}=-m_{1} m_{2} \\
& \alpha_{2}=k_{F} m_{1}+k_{1} m_{2}+k_{F} m_{2}-k_{N} m_{2} \\
& \alpha_{3}=k_{N}{ }^{2}-k_{1} k_{F}-k_{F} k_{N}
\end{aligned}
$$

This represents a fourth order polynomial without odd terms, which therefore is symmetric. Due to its symmetry, the equation has two positive real roots that are mirrored around the y-axis, and can be expressed in its factored form as,

$$
y=-m_{1} m_{2}\left(\omega-\omega_{1}\right)\left(\omega-\omega_{2}\right)\left(\omega+\omega_{1}\right)\left(\omega+\omega_{2}\right)
$$

After expansion this equation yields,

$$
y=\omega^{4} \beta_{1}+\omega^{2} \beta_{2}+\beta_{3}
$$

with,

$$
\begin{aligned}
& \beta_{1}=-m_{1} m_{2} \\
& \beta_{2}=m_{1} m_{2}\left(\omega_{1}{ }^{2}+\omega_{2}{ }^{2}\right) \\
& \beta_{3}=-m_{1} m_{2} \omega_{1}{ }^{2} \omega_{2}{ }^{2}
\end{aligned}
$$


A comparison of the coefficients of (17) and (20) yields the following equations,

$$
\begin{aligned}
k_{2} m_{1}+k_{N} m_{1}+k_{1} m_{2}+k_{2} m_{2} & =m_{1} m_{2}\left(\omega_{1}^{2}+\omega_{2}^{2}\right) \\
k_{1} k_{2}+k_{1} k_{N}+k_{2} k_{N} & =m_{1} m_{2} \omega_{1}^{2} \omega_{2}{ }^{2}
\end{aligned}
$$

In order to be dynamically stable, the systems eigenfrequencies have to be real and positive,

$$
\omega_{1}>0 ; \quad \omega_{2}>0
$$

This necessitates the right-hand sides of (21) and (22) to be greater than 0 , and therefore, two conditions for $k_{N}$ can be elaborated,

$$
k_{N}>-\frac{k_{F} m_{1}+k_{1} m_{2}+k_{F} m_{2}}{m_{1}}
$$

and,

$$
k_{N}>\frac{k_{F}}{2}-\sqrt{\frac{k_{F}^{2}}{4}+k_{1} k_{F}}
$$

A parametric study, which is omitted in the interest of brevity here, has shown that the latter condition is stricter than the former one, and therefore, is governing for the design. For the remainder of this work, the maximum negative stiffness of the NSE will be determined relative to the maximal value of eq. 25 in percent \%.

\section{Band gaps and wave propagation}

On the one hand the effect of the NSE on the band gap behaviour is expected to be advantageous due to the amplification force, while on the other hand the effect of its inevitable nonlinearity is yet unknown. In this section the potential band gaps of the system will be investigated for the linearized as well as the elastic non-linear structure with parameters corresponding to the FULL system.

\subsection{Band gaps of the linear system}

Based on the multi-layered foundation depicted in Figure 5(b), a chain of unit cells endowed with NSEs can be established as shown in Figure 6 where 
$u$ denotes the displacement of the discretized mass with the subscript $(1,2)$ determining the mass and the superscript $(j-1, j, j+1)$ defining the relative location of the unit cell. Note that for the linear case the NSE is represented by a linear negative spring with value $k_{N}$ from Eq. (8). Under the aid of the Floquet-Bloch theorem [36] it becomes possible to relate the movement of the previous and subsequent unit cells, to the unit cell under study with,

$$
\mathbf{u}(x, t)=\mathbf{u}_{\mathbf{0}} e^{i(\mathbf{q} \mathbf{x}-\omega t)}
$$

Here, $\mathbf{u}(x, t)$ denotes the displacement vector at position $\mathbf{x}$ at time $t, \mathbf{u}_{\mathbf{0}}$ the amplitude of the displacement at the reference position $(\mathbf{x}=0), \omega$ the frequency of the propagating wave, and $\mathbf{q}$ the wave vector. For a $1 \mathrm{D}$ system the wave vector becomes a scalar and is defined as the inverse of the wavelength ( $q=$ $1 / \lambda)$. Furthermore, the distance between the cells will be set to unity, therefore reducing the position vector $\mathbf{x}$ to a scalar of \pm 1 and the range of $q$ to $-\pi \leq q \leq \pi$. It is now possible to write the boundary conditions in the frequency domain for a discretized chain with,

$$
u_{1,2}^{j \pm 1}=u_{1,2} e^{ \pm i q}
$$

This condition can be applied to the EOMs of a typical periodic unit cell which read,

$$
\begin{aligned}
m_{1} \ddot{u}_{1}^{j}+\left(2 k_{1}+k_{F}\right) u_{1}^{j}-k_{1} u_{1}^{j+1}-k_{1} u_{1}^{j-1}-\left(k_{F}-k_{N}\right) u_{2}^{j} & =0 \\
m_{2} \ddot{u}_{2}^{j}-\left(k_{F}-k_{N}\right) u_{1}^{j}+k_{F} u_{2}^{j}-k_{N} u_{1}^{j-1} & =0
\end{aligned}
$$

After the application of the boundary condition (28) to (29), the discrete eigenvalue problem can be formulated with,

$$
\left(\mathbf{K}-\omega^{2} \mathbf{M}\right) \mathbf{u}=0
$$

The non-trivial solution for this problem yields the dispersion relation,

$$
\begin{aligned}
& \operatorname{Cos}(q)= \\
& \frac{2 k_{1} k_{F}+2 k_{F} k_{N}-2 k_{N}^{2}+\left(-k_{F} m_{1}-2 k_{1} m_{2}-k_{F} m_{2}\right) \omega^{2}+m_{1} m_{2} \omega^{4}}{2\left(-k_{1} k_{F}-k_{F} k_{N}+{k_{N}}^{2}\right)+2 k_{1} m_{2} \omega^{2}}
\end{aligned}
$$


which sheds light on the wave propagation behaviour. For the sake of demonstration, the frequency of the resonator shall be set to $3 \mathrm{~Hz}$, while all other structural values match the FULL system from Table 2. The dispersion is depicted in Figure 7(a) for varying NSE values, with the percentage indicating the relationship of the applied NSE to the maximal allowable negative stiffness value from (25). Here the maximum values of the acoustic branches are highlighted with dotted lines, while also the minimum value of the optical branch is marked with a dotted line, in order to highlight the band gap. When observing the dispersion branches, it becomes clear that an increase in negative stiffness entails a downwards shift of the band gaps lower bound, i.e. the acoustic branch, while the upper bound, i.e. optical branch, remains unchanged. Clearly, this is related to the shift of Eigenfrequencies in the system, which can be attributed to the softening effect of the NSE. Furthermore, Figure 7(b) depicts the frequency response function FRF of the system with $75 \%$ of the maximal allowable NSE for 1, 10, and 100 Layers. The reader may note that for the constellations of 1 , and 10 Layers, the attenuation zone stretches further than the band gap predicts, which is a property that cannot be found in classical metamaterials and is most likely due to the assembly of the springs. More precisely, the resonators are connected not only to the slab they are intended to act upon, but also, by means of the NSE, to the previous slab.

\subsection{Band gaps in the nonlinear system}

In this subsection, the effect of nonlinearity on the band gap behaviour is discussed. More precisely, the HBM will be applied to the unit cell formulation of the system of EOMs and a dispersion relation, dependent on the nonlinearity of the system, established. Note that this procedure will provide an approximate but analytical solution for the nonlinear band gap problem, where the Floquet-Bloch theorem is not strictly applicable. However, some studies have shown that periodic structures with weak nonlinearities can propagate Blochlike waves with one dominant component and can, therefore, be analysed by means of standard techniques [37. As a result, the HBM can be applied to find 


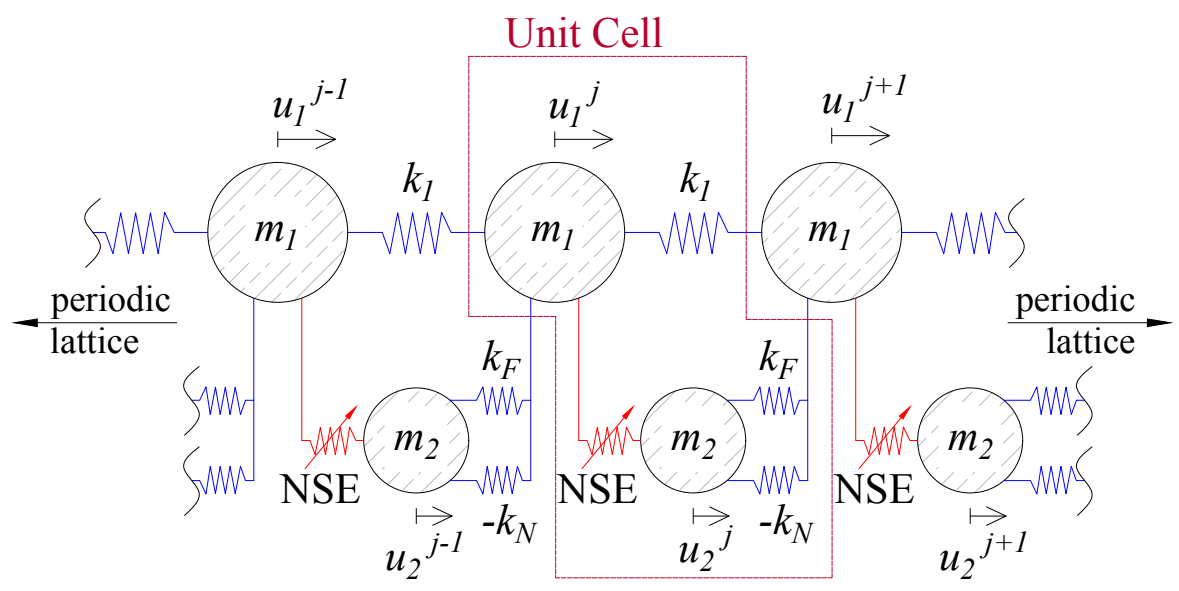

Figure 6: Infinite lattice and unit cell depiction.
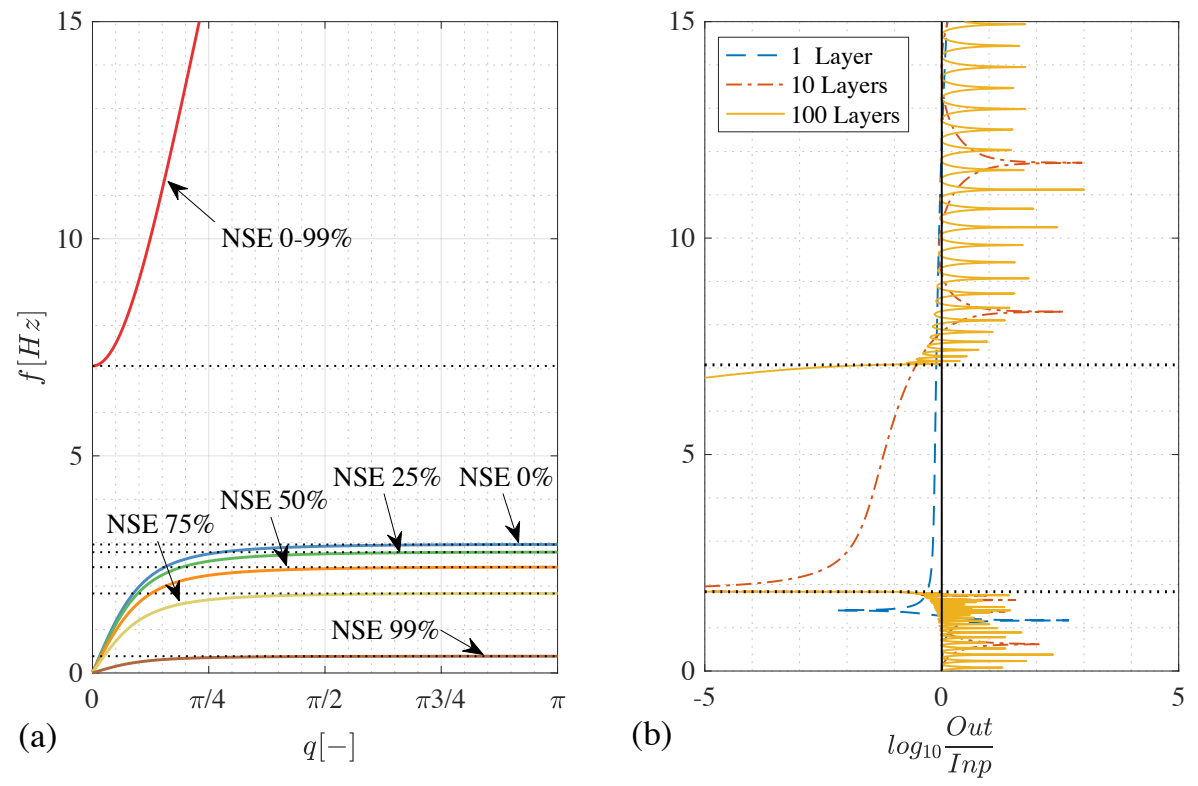

Figure 7: Wave propagation behaviour of the linear system: (a) Dispersion relation of the unit cell for varying NSE values (acoustic branch $=\mathrm{AB}$ ); (b) FRF of the linear system for 1 , 10, and 100 Layers with an NSE value of $75 \%$. 
Bloch wave compatible solutions when coupled with the Floquet 1D boundary conditions [28, 38]. Additionally, THAs will be run for different excitation frequencies and amplitudes, in order to obtain an amplitude dependent FRF, which subsequently can be compared to the results of the band gap analysis.

\subsubsection{Analytical evaluation of nonlinear band gaps}

In order to apply the HBM, we first need to define the EOMs of the nonlinear system. For the sake of simplicity, the displacement of the resonator is defined as relative to the main mass and is denoted with $v^{x}$, where $x \in\{j-1, j, j+1\}$ denotes the relative unit cell. The EOMs read,

$$
\begin{array}{r}
m_{1} \ddot{u}_{1}^{j}+\left(2 k_{1}+k_{F}\right) u_{1}^{j}-k_{1} u_{1}^{j-1}-\left(k_{F}-k_{N}\right)\left(u_{1}^{j-1}+v^{j-1}\right)-k_{1} u_{1}^{j+1}-f=0 \\
m_{2}\left(\ddot{u}_{1}^{j}+\ddot{v}^{j}\right)+\left(k_{F}-k_{N}\right)\left(u_{1}^{j}+v^{j}\right)+u_{1}^{j+1}\left(k_{F}-k_{N}\right)+f=0
\end{array}
$$

Here, $f$ describes the nonlinear force, which will be simplified with (4) (7) and (9) to read,

$$
f(t)=a_{N S E} v(t)+b_{N S E} v(t)^{3}
$$

This system is reminiscent of a duffing oscillator. Note however, that the resonators are connected to the unit cells above and below them, therefore, representing a new type of resonator chain. The general formulation of the harmonic balance method with complex exponentials can be written as,

$$
y(t)=\sum_{n=1}^{\infty} Y_{n} e^{i n \omega t}+\bar{Y}_{n} e^{-i n \omega t}
$$

with $y(t)$ being the motion of a generic degree of freedom in time, $n$ denotes the

harmonic, while $Y_{n}$ and $\bar{Y}_{n}$ are the complex and complex conjugate amplitudes of the complex exponential series. Conveniently, the Floquet-Bloch boundary condition can be applied to this formula as follows,

$$
y^{j \pm 1}(t)=\sum_{n=1}^{\infty}\left(Y_{n} e^{i n \omega t}+\bar{Y}_{n} e^{-i n \omega t}\right) e^{ \pm i q_{n}}
$$


with $q_{n}$ being the wave number for harmonic $n$. Note that this formulation is equivalent to the formulation for the linear system and assumes a resonator mass chain with unitary distance between unit cells. This method can now be truncated for the first harmonic $(n=1)$ yielding,

$$
\begin{aligned}
& u^{j \pm 1}(t)=\left(U_{1} e^{i \omega t}+\bar{U}_{1} e^{-i \omega t}\right) e^{ \pm i q_{1}} \\
& v^{j \pm 1}(t)=\left(V_{1} e^{i \omega t}+\bar{V}_{1} e^{-i \omega t}\right) e^{ \pm i q_{1}}
\end{aligned}
$$

Here $U_{1}, \bar{U}_{1}, V_{1}$ and $\bar{V}_{1}$, denote the complex and complex conjugate amplitudes of the harmonic motion of $u(t)$ and $v(t)$, respectively. Subsequently these expressions must be applied to the EOMs $(32)$ and (33) and their harmonics balanced, by equating the coefficients in front of $e^{i \omega t}$ with 0 , which yields the following set of equations,

$$
\begin{aligned}
& -\left(U_{1}+U_{1}\left(k_{F}-k_{N}\right)+V_{1}\left(k_{F}-k_{N}\right)\right) e^{-i q_{1}}-U_{1} k_{1} e^{i q_{1}}-a_{N S E} V_{1} \\
& -3 b_{N S E} V_{1}^{2} \bar{V}_{1}+2 U_{1} k_{1}+U_{1}\left(k_{F}-k_{N}\right)-U_{1} m_{1} \omega^{2}=0 \\
& \quad-U_{1}\left(k_{F}-k_{N}\right) e^{i q_{1}}+a_{N S E} V_{1}+3 b_{N S E} V_{1}^{2} \bar{V}_{1}+U_{1}\left(k_{F}-k_{N}\right) \\
& \quad+V_{1}\left(k_{F}-k_{N}\right)-\left(U_{1} m_{2}+U_{1} m_{2}\right) \omega^{2}=0
\end{aligned}
$$

After the substitution of (40) in (39), $U_{1}$ can be eliminated and the following dispersion relation can be obtained,

$$
\cos \left(q_{1}\right)=\frac{N}{D}
$$

with the numerator $\mathrm{N}$ and the denominator $\mathrm{D}$ being equal to,

$$
\begin{aligned}
N= & 3 b_{N S E} V_{1} \bar{V}_{1}\left[2 k_{1}+2\left(k_{F}-k_{N}\right)-\left(m_{1}+m_{2}\right) \omega^{2}\right] \\
& +a_{N S E}\left[2 k_{1}+2\left(k_{F}-k_{N}\right)-\left(m_{1}+m_{2}\right) \omega^{2}\right] \\
& -\omega^{2}\left[\left(k_{F}-k_{N}\right)(m 1+m 2)+2 k_{1} m_{2}\right] \\
& +2 k_{1}\left(k_{F}-k_{N}\right)+\omega^{4} m_{1} m_{2}
\end{aligned}
$$

and,

$$
\begin{aligned}
D= & 6 b_{N S E} V_{1} \bar{V}_{1}\left[k_{1}+k_{F}-k_{N}\right] \\
& +2 a_{N S E}\left[k_{1}+k_{F}-k_{N}\right] \\
& +k_{1}\left[k_{F}-k_{N}-m_{2} \omega^{2}\right]
\end{aligned}
$$




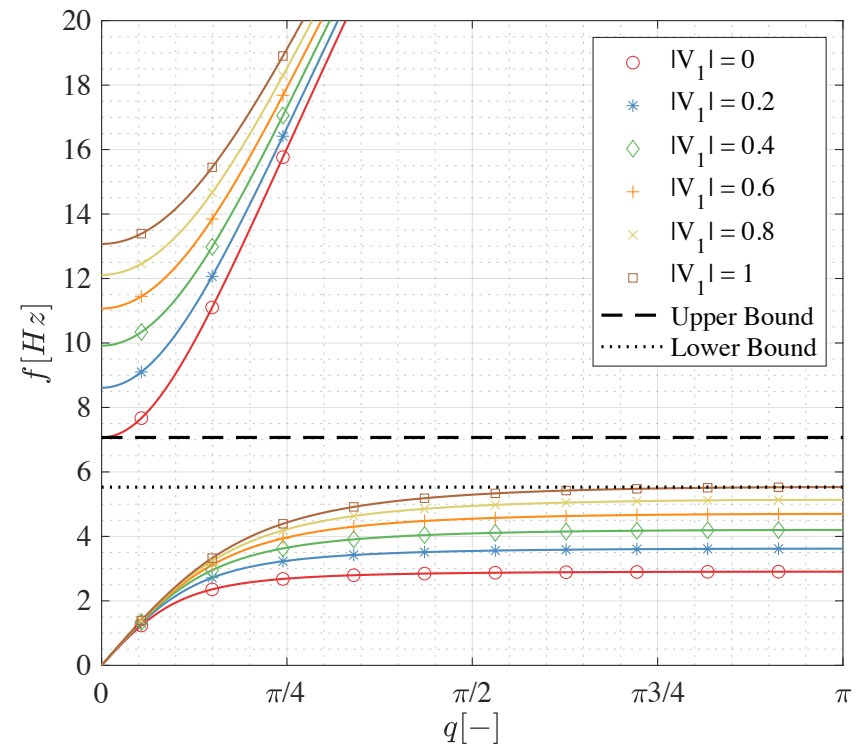

Figure 8: Dispersion diagram of the non-linear FULL system with varying amplitudes and $75 \%$ of the maximum NSE value.

The new dispersion relation is dependent on $b_{N S E} V_{1} \bar{V}_{1}$, which represents the nonlinearity of the system, and is plotted dependent on the amplitude $\left|V_{1}\right|=$ ${ }_{255} \sqrt{V_{1} \bar{V}_{1}}$, but for a constant $b_{N S E}$ in Figure 8 . Here, the dispersion branches move to a higher frequency spectrum since an increase of nonlinearity entails a higher participation of $b_{N S E}$, which in turn causes a stiffening of the system. The upwards shift of the dispersion branches may seem to indicate that the band gap shifts towards higher frequencies and that the band gap widens, as has been found for classical duffing oscillator type metamaterials [28, 29. However, when waves that fall within the band gap of a specific level of nonlineartiy are attenuated, their change in intensity subsequently changes the level activated nonlinearity and therefore also the resulting band gap of the system. Based on this we conclude that only the common band gap of the linear and nonlinear system can be retained, which entails that the frequency range narrows towards the upper bound with an increase in nonlinearity. This is highlighted with the dotted line for the lower bound and the dashed line for the upper bound in 
Figure 8 and will be illustrated more clearly with the simulations in the time domain conducted in the next section.

The reader may note that Figure 8 shows amplitudes up to $1 \mathrm{~m}$ relative displacement, which is not possible for the geometry shown earlier. However, the motion range of the resonators is restricted only by the column spacing, which in turn can easily be changed in order to achieve a highly nonlinear range. Therefore, amplitudes up to $1 \mathrm{~m}$ are considered to clearly show the trend of the dispersion branches. Finally, while a relative displacement of $1 \mathrm{~m}$ may not seem realistic, both the dispersion and wave propagation analysis display possible effects that the nonlinear NSE entail on a periodic system.

\subsubsection{FRF of the nonlinear system}

In order to evaluate the FRF of the nonlinear system, an iterative time integration procedure was implemented based on a Newton-Raphson solver inserted into an implicit Newmark beta scheme. The equations of motion for the system read,

$$
\mathbf{M} \ddot{\mathbf{u}}(t)+\mathbf{C} \dot{\mathbf{u}}(t)+\mathbf{K u}(t)+\mathbf{R}(\mathbf{u}, t)=\mathbf{F}(t)
$$

where $\mathbf{M}, \mathbf{C}$, and $\mathbf{K}$ are the linear mass, damping and stiffness matrices, which can be written for the system displayed in Figure 5(b) with an arbitrary number of layers. Note that the damping matrix $\mathbf{C}$ contains Rayleigh damping of $3 \%$ between 2 and $8 \mathrm{~Hz}$ for the system without resonators, in order to attenuate the vibration of the eigenmodes induced by the transient nature of the simulation. Note that the actual proportional damping of the complete system is even lower, due to the added mass and stiffness of the resonators, which were not taken into account when constructing the damping matrix. This further provides the band gap range with low damping values, while the resonators remain undamped, which minimizes the effect that the overall damping has on the band gap phenomenon. Moreover, $\mathbf{R}(\mathbf{u}, t)$ denotes the vector containing the nonlinear restoring forces, deriving from the NSE i.e. eqs. (4) or (7), while the forcing term $\mathbf{F}(t)$ can be rewritten as $\mathbf{M I} \ddot{u}_{g}(t)$, since the EOMs were formulated in terms of relative ground displacement. A harmonic ground acceleration for 
every frequency can be obtained with,

$$
\ddot{u}_{g}(t)=A e^{i \omega_{g} t}
$$

with $A$ being the amplitude of the wave and $\omega_{g}$ being the radial frequency of the excitation. Integrate twice over time and a harmonic ground displacement can be obtained with,

$$
u_{g}=-\omega_{g}^{2} A e^{i \omega_{g} t}
$$

In order to obtain an FRF, a finite chain of masses and resonators is subjected to this harmonic excitation on one side (the ground) and its absolute displacement response, once the steady state is reached, recorded on the other side. For these simulations the true nonlinearity and the polynomial approximation were considered for 1, 10 and 20 layers, without the presence of a superstructure and an NSE level of $25 \%$ of the maximum. In Figures 9 (a), (b), (c), (d), (e) and (f) the FRFs are displayed with the frequency on the x-axis, the amplitude of excitation in meters on the y-axis, and the absolute displacement response of the top layer relative to the excitation frequency on the z-axis. Furthermore, a gray horizontal plane is drawn at unity, where the response at the top is equal to the input at the bottom, which highlights the attenuation of the excitation. If the response is smaller than unity, the signal is being attenuated and a band gap can be expected. Note that the first mode of the complete 20 layered system (including the resonators) was located at $\omega_{1}=3.68 \mathrm{rad} / \mathrm{s}$ at a modal damping value of $\zeta_{1}=1.4 \%$, which resulted in a settling time of $t_{S}=4 /\left(\zeta_{1} \omega_{1}\right)=80 \mathrm{~s}$. Therefore a simulation length of 100 seconds was chosen.

For low excitations the structure remains in the linear range, which entails that the numerical FRF resembles the linear FRF for the lowest amplitude. When observing the FRFs for the true nonlinear system, an area without convergence can be seen in the high frequency, high excitation, region, due to the infinite stiffness at $v \rightarrow l$. Furthermore, when comparing the true nonlinear system to the polynomial approximation, it becomes clear that the approximation yields very similar results, even in the highly nonlinear range, where higher harmonics start to appear. This becomes particularly evident for the one layered 
system where a clear upwards shift of the eigenfrequency can be observed and a second spike representing a higher harmonic appears at 3 times the value of the systems first eigenfrequency. Furthermore, as discussed in the previous section, the upper bound of the band gap, or in the finite case the attenuation zone, does not shift upwards with an increase in excitation amplitude (activation of nonlinearity). Instead, Figure 9 depicts clearly that the upper bound shows only a slight upwards shift, due to the finiteness of the system, while the lower bound approaches the upper bound with increased nonlinearity. Moreover, with more layers the system tends to show a better attenuation within the band gap, which is due the added layers, but also due to the necessary damping present in the systems. From these results and the nonlinear dispersion analysis from the previous section, we conclude that with an increase of excitation amplitude the band gap will gradually disappear towards the upper bound.

However, for the system to introduce resonance in the higher harmonics, some of the wave energy must be shifted away from the primary modes, which will be discussed with further THAs in Section 5 With respect to the band gap behaviour on the other hand, the effects of the nonlinearity are clearly detrimental.

\section{Optimization of the coupled system}

As shown in previous publications [4] and [13], once the Metafoundation is coupled to a superstructure, the complete coupled system needs to be optimized. For this reason, we propose an optimization algorithm herein, based on calcula-

325 tion in the frequency domain, which represents a simplification of the algorithm established in [13] and depends on the structure and the ground motion.

\subsection{Ground motion models}

To narrow down the expected vibrations for the structure at hand, we chose to place it at a seismic prone site in Italy, namely Priolo Gargallo, and charac330 terized it with a uniform hazard spectrum (UHS). The red solid line in Figure 

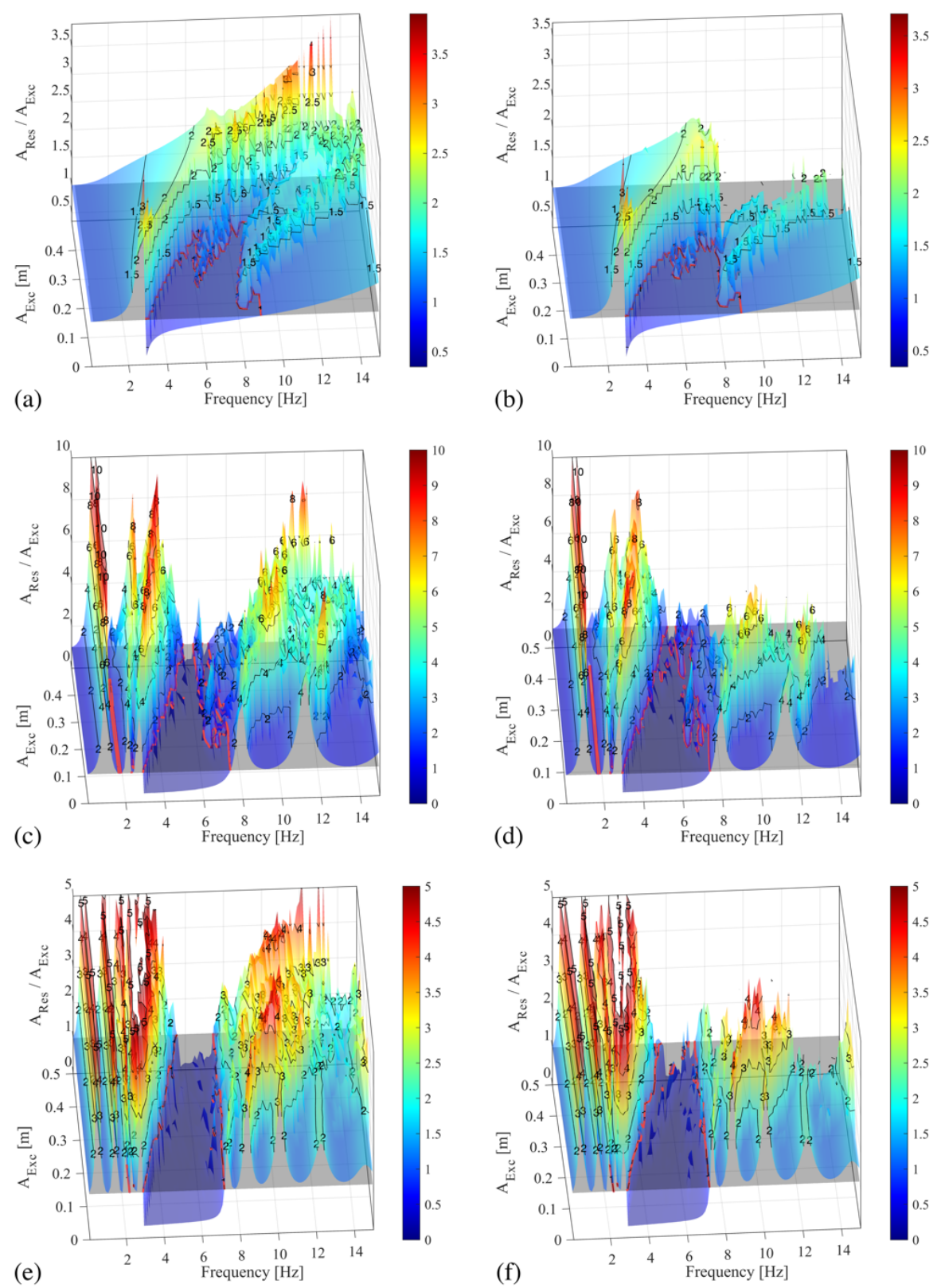

Figure 9: Numerical FRF for the FULL system with 25\% NSE: (a) 1 layer and polynomial nonlinearity; (b) 1 layer and true nonlinearity; (c) 10 layers and polynomial nonlinearity; (d) 10 layers and true nonlinearity; (e) 20 layers and polynomial nonlinearity; (f) 20 layers and true nonlinearity. 

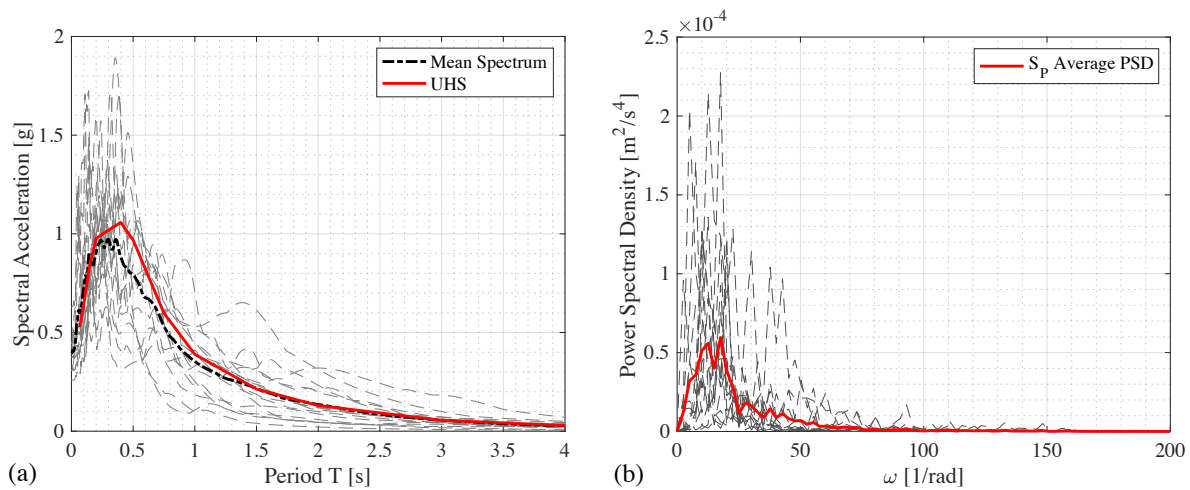

Figure 10: Ground motion spectra: (a) Response spectra including the UHS and the mean response spectrum; (b) PSDs of all ground motion records and their average estimated with Welch's method.

10 shows the UHS of Priolo Gargallo for a return period of 475 years, which can be fitted with real records of ground motions (Figure 10(a) dashed grey lines). Here, the average response spectrum of the seismic events, see Figure 10(a) black dashed-dotted line, was fitted to the UHS in a least square sense. and are listed in Table 3 .

In order to obtain the power spectral density (PSD) of the selected records, which can be used in the optimization algorithm, Welch's method was applied with the following parameters: 8000 data points per seismic record, 1000 data points per finite section of the signal, 500 data points overlap, and a standard Hamming window. The resulting average PSD for each individual record (dashed grey lines) and the total average over all records (solid red line) are displayed in Figure 10(b).

\subsection{Optimization algorithm}

Given the epistemic and aleatoric uncertaintity of the seismic input, it is not feasible to tackle an optimization problem of a system endowed with linear or nonlinear devices on a conventional time basis. Therefore, a more accurate probabilistic (stochastic) approach is required in which both the excitation and 
Table 3: List of ground motion records.

\begin{tabular}{llrrr}
\hline Event & ID & M & RJb [km] & PGA [m/s $\left.{ }^{2}\right]$ \\
\hline Loma Prieta & BRN090 & 6.93 & 3.85 & 0.4067 \\
Kalamata & 000414ya & 5.9 & 11 & 0.3738 \\
South Iceland & 004673ya & 6.5 & 15 & 0.4224 \\
L'Aquila Mainshock & IT0792ya & 6.3 & 4.8698 & 0.6287 \\
Friuli 2nd Shock & IT0078ya & 5.6 & 26.2079 & 0.4023 \\
Northridge-01 & ORR360 & 6.69 & 20.11 & 0.3749 \\
Umbria Marche & 000594ya & 6 & 11 & 0.4224 \\
Montenegro & 000199ya & 6.9 & 16 & 0.3071 \\
Erzincan & 000535ya & 6.6 & 13 & 0.4224 \\
Friuli Italy-01 & A-TMZ270 & 6.5 & 14.97 & 0.2585 \\
South I. (aftershock) & 006328ya & 6.4 & 12 & 0.3914 \\
Ano Liosia & 001715ya & 6 & 14 & 0.3103 \\
L'Aquila Mainshock & IT0789ya & 6.3 & 4.6338 & 0.4024 \\
L'Aquila Mainshock & IT0790ya & 6.3 & 4.3919 & 0.4459 \\
L'Aquila Mainshock & IT0791ya & 6.3 & 5.6501 & 0.3300 \\
\hline
\end{tabular}


the response are described in terms of statistical parameters such as the mean square, - that is null for seismic excitations- and the variance of vibration amplitudes. As a result, the herein proposed approach heavily relies on random vibration [39] and optimizes the foundation under consideration of the superstructure and the ground motion. The first step is to formalize the system of Equations Of Motion (EOMs) with,

$$
\mathbf{M} \ddot{\mathbf{u}}(t)+\mathbf{C} \dot{\mathbf{u}}(t)+\mathbf{K u}(t)=\mathbf{M I} \ddot{u}_{g}(t)
$$

The mass, stiffness and damping matrices, $\mathbf{M}, \mathbf{K}$ and $\mathbf{C}$ can be constructed for the dynamic system displayed in Figure 5 (a) with the values of Table 2 and the indication about the damping given in the text, while $\mathbf{I}$ represents the identity vector. Note that for the optimization procedure, the negative stiffness enters as a linear spring, since the computations in the frequency domain demand linearity. Firstly, we multiply the EOMs with $e^{-i \omega t}$ and integrate over time to obtain,

$$
\int_{-\infty}^{+\infty}[\mathbf{M} \ddot{\mathbf{u}}(t)+\mathbf{C} \dot{\mathbf{u}}(t)+\mathbf{K u}(t)] e^{-i \omega t} d t=\int_{-\infty}^{+\infty} \mathbf{M I} \ddot{u}_{g}(t) e^{-i \omega t} d t
$$

Subsequently, the response and excitation are transformed into the frequency domain via a Fourier transform with,

$$
\begin{aligned}
& \mathcal{U}(\omega)=\int_{-\infty}^{+\infty} \mathbf{u}(t) e^{-i \omega t} d t \\
& \mathcal{F}(\omega)=\int_{-\infty}^{+\infty} \mathbf{M I} \ddot{u}_{g}(t) e^{-i \omega t} d t
\end{aligned}
$$

where the derivatives of the response can be obtained with,

$$
\begin{aligned}
& \int_{-\infty}^{+\infty} \dot{\mathbf{u}}(t) e^{-i \omega t} d t=i \omega \int_{-\infty}^{+\infty} \mathbf{u}(t) e^{-i \omega t} d t=i \omega \mathcal{U}(\omega) \\
& \int_{-\infty}^{+\infty} \ddot{\mathbf{u}}(t) e^{-i \omega t} d t=-\omega^{2} \int_{-\infty}^{+\infty} \mathbf{u}(t) e^{-i \omega t} d t=-\omega^{2} \mathcal{U}(\omega)
\end{aligned}
$$

With expressions 49 - 52 the system can be reformulated in the frequency domain as,

$$
\left(-\omega^{2} \mathbf{M}+i \omega \mathbf{C}+\mathbf{K}\right) \mathcal{U}(\omega)=\mathcal{F}(\omega)
$$


Here, the transmission matrix can be defined and parametrized as,

$$
\mathcal{H}\left(\omega, k_{F}, c_{R}\right)=\left[-\omega^{2} \mathbf{M}+i \omega \mathbf{C}\left(c_{R}\right)+\mathbf{K}\left(k_{F}\right)\right]^{-1}
$$

where $k_{F}$ is a variable in the stiffness matrix $\mathbf{K}$, and $c_{R}$ represents a viscous damper, which works in parallel with $\left(k_{F}-k_{N}\right)$, in the damping matrix C. Note that the frequency of the resonator can be approximated with $\omega_{R}=\sqrt{k_{F} / m_{2}}$, while its damping can be represented with the critical damping ratio $\zeta_{R}=c_{R} / 2 \sqrt{\left(k_{F}-k_{N}\right) m_{2}}$. With these expressions and the definition of the approximate Power Spectral Density (PSD) of the response given by [40] we can rewrite the system response in terms of PSD as,

$$
\mathbf{S}_{\mathcal{U}}\left(\omega, \omega_{R}, \zeta_{R}\right)=\left|\mathcal{H}\left(\omega, \omega_{R}, \zeta_{R}\right)\right|^{2} \mathbf{S}_{g}(\omega)
$$

where $\mathcal{H}\left(\omega, \omega_{R}, \zeta_{R}\right)$ denotes the transmission matrix as defined in eq. (54) and $\mathbf{S}_{g}(\omega)$ describes the PSD of the excitation $\mathcal{F}(\omega)$, which is comprised of the average PSD obtained in Section 4.1. multiplied with MI. Furthermore, with the transformation by Wiener-Khintchine [40, the variance of a signal can be calculated based on the relationship of the autocorrelation function with the PSD as,

$$
\sigma^{2}=R(0)=\int_{-\infty}^{+\infty} S(\omega) d \omega
$$

here, $R(0), S(\omega)$, and $\sigma_{u}^{2}$ denote the autocorrelation function, the PSD, and the variance of the response, respectively. In line with this, the relative displacement of the impulsive mode and the top slab can be estimated with,

$$
\sigma_{r e l}^{2}=\int_{-\infty}^{+\infty}\left(S_{j}\left(\omega, \omega_{R}, \zeta_{R}\right)-S_{k}\left(\omega, \omega_{R}, \zeta_{R}\right)\right) d \omega
$$

where, $S_{j}\left(\omega, \omega_{R}, \zeta_{R}\right)$ and $S_{k}\left(\omega, \omega_{R}, \zeta_{R}\right)$ are the PSDs of the relative ground displacements of the jth and kth degree of freedom (DOF), while $\sigma_{\text {rel }}^{2}$ represents the variance of the relative displacement between those DOFs. Hence, we formulate the Performance Index $P I\left(\omega_{R}, \zeta_{R}\right)$ as,

$$
P I\left(\omega_{R}, \zeta_{R}\right)=\frac{\sigma_{M E T A}^{2}\left(\omega_{R}, \zeta_{R}\right)}{\sigma_{T R A D}^{2}}
$$

where, $\sigma_{M E T A}^{2}$ and $\sigma_{T R A D}^{2}$ denote the variance of the relative drift of the impulsive mode for a system with and without Metafoundation, respectively. 


\section{Behaviour of the coupled system}

\subsection{Behaviour of the system in the frequency domain}

When running the optimization procedure on the coupled system subjected to the average PSD of the earthquakes, the PI can be computed and plotted for various frequency and damping ratios for the resonators, as displayed in Figures 11(a), (b), (c), and (d). Here, $f_{R}$ corresponds to the optimal frequency of the resonators, while $\zeta_{R}$ is the optimal damping ratio of the resonators, which are computed for the Metafoundation with $0 \%, 25 \%, 50 \%$, and $99 \%$ of the maximal admissible NSE value, obtained from eq. (25). Note that a change in frequency allows for a recalculation of the maximal value of $k_{N}$, which would entail an iteration of the optimization scheme for every increase in frequency. This would multiply the computational effort by the amount of iterations necessary to find the final value, and has therefore been omitted in the interest of efficiency. Note further that the actual applicable NSE value is most likely determined by construction requirements, hence smaller than the theoretically possible one. Instead, the initial guess of $k_{F}$ was chosen to produce a frequency of $3 \mathrm{~Hz}$ and used to determine the maximal admissible NSE value.

As shown in Figure 11 the value of the PI decreases with an increase in NSE value, while the optimal tuning frequency and damping ratio of the resonator have a clear effect on the PI. Table 4 summarizes the optimal parameters for all investigated systems and displays that the optimal value of the stiffness tends to increase for high NSE values in the REDUCED and MINIMAL systems, while for the FULL system it decreases first and then increases again. This is most likely due to the NSE changing the frequency of the resonators, as can be observed in Figure 7 and the NSE reducing the overall stiffness, thereby shifting the critical excitation frequency of the tank. From this tendency we conclude that an optimization, as carried out herein, is necessary for the design of a negative stiffness endowed system and that the superstructure should not

375 be neglected. Furthermore, it is worth noting that the surface becomes flatter with an increase in NSE value, which entails that for higher NSE values the 
system becomes less dependent on the optimal tuning of the resonators. This effect is particularly interesting for the frequency of the resonator, as a precise tuning can be challenging in a real-life application. A similar trend can be observed for the damping ratio, where the optimal values are located at very high damping ratios of $19 \%$ to $30 \%$, while only small improvements in terms of PI can be obtained above a value of $10 \%$.

Table 4: Results from the optimization for the relevant Metafoundation setups.

\begin{tabular}{l|ccc|ccc|ccc}
\hline NSE & \multicolumn{3}{|c|}{ FULL } & \multicolumn{3}{c|}{ REDUCED } & \multicolumn{3}{c}{ MINIMAL } \\
$k_{N \max }$ & $f_{R}$ & $\zeta_{R}$ & $P I$ & $f_{R}$ & $\zeta_{R}$ & $P I$ & $f_{R}$ & $\zeta_{R}$ & $P I$ \\
\hline 0 & 4.00 & 0.19 & 0.502 & 4.75 & 0.11 & 0.62 & 5.85 & 0.03 & 0.882 \\
25 & 3.70 & 0.23 & 0.388 & 4.85 & 0.14 & 0.496 & 5.95 & 0.04 & 0.726 \\
50 & 3.55 & 0.24 & 0.286 & 4.90 & 0.17 & 0.407 & 6.10 & 0.06 & 0.617 \\
75 & 3.95 & 0.27 & 0.211 & 4.80 & 0.19 & 0.332 & 6.25 & 0.07 & 0.530 \\
99 & 4.50 & 0.30 & 0.161 & 5.00 & 0.20 & 0.265 & 6.45 & 0.08 & 0.462 \\
\hline
\end{tabular}

\subsection{System response in the time domain}

From the optimal values determined in the previous section, the stiffness of spring $k_{F}$ and the damping value of $c_{R}$ can be evaluated from the expressions $k_{F}=\omega_{R}^{2} m_{2}$ and $c_{R}=\zeta_{R} 2 \sqrt{\left(k_{F}-k_{N}\right) m_{2}}$. Furthermore, the length of the compression member was chosen beforehand and is shown in Table 1, while the Prestress force in the compression member can be obtained from the relationship $a_{N S E}=-P / l$ where $a_{N S E}=k_{N}$ with,

$$
P=-k_{N} l
$$

Besides this, the nonlinearity parameter $\epsilon$ yields realistic values for $k_{P}$ when set to 0.95 , where $k_{P}$ can be evaluated form (10) as,

$$
k_{p}=\frac{P}{(1-\epsilon) l}
$$

With these parameters and the optimal values found from the optimization, summarized in Table 4 , the complete nonlinear system can be constructed and 

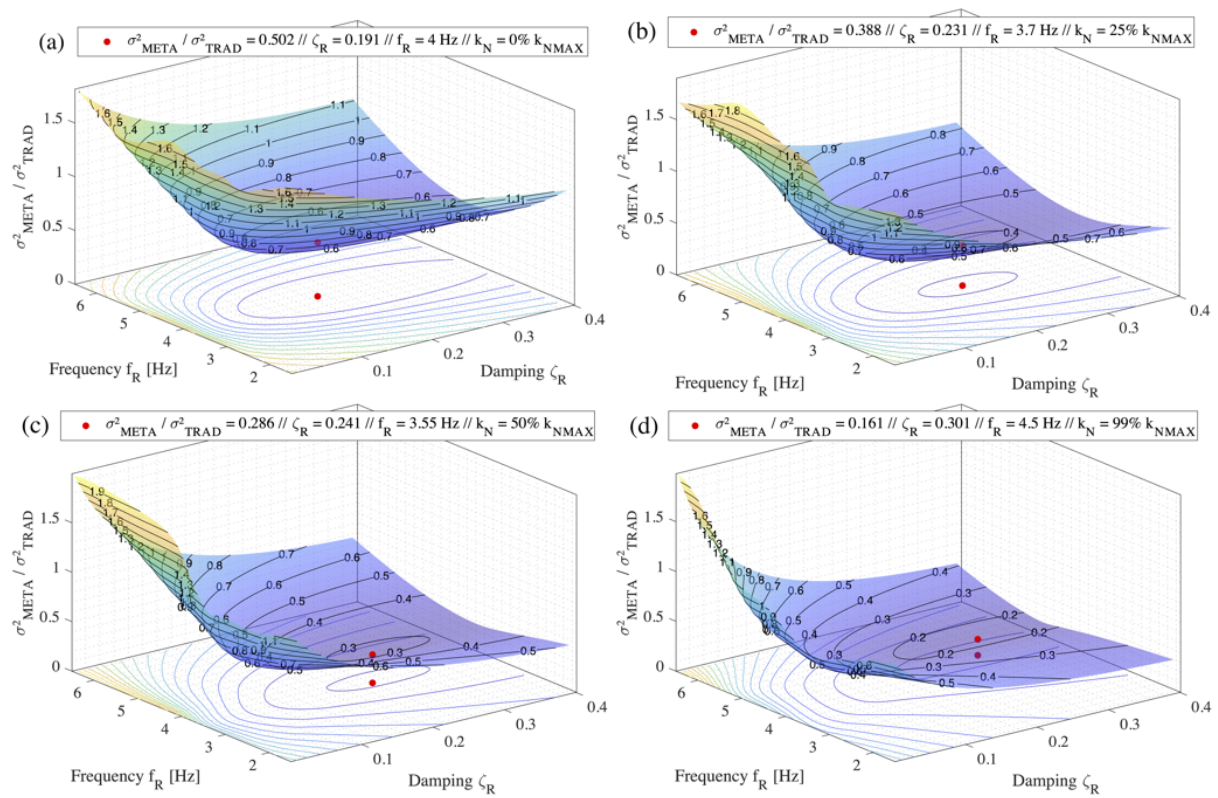

Figure 11: Optimization surface plots for: (a) PI for the FULL system with $0 \% k_{N} \max$; (b) PI for the FULL system with $25 \% k_{N \max }$; (c) PI for the FULL system with $50 \% k_{N \max }$; (d) PI for the FULL system with $99 \% k_{N \max }$. 
subjected to the ground motions from Table 3 For the sake of comparison, various Metafoundation layouts were considered and the resulting base shear developments of the respective tank evaluated with,

$$
V(t)=\left[u_{i}(t)-u_{1}(t)\right] k_{i}+\left[u_{c}(t)-u_{1}(t)\right] k_{c}
$$

where, $V(t), u_{i}(t), k_{i}, u_{c}(t), k_{c}$, and $u_{1}(t)$ are the base shear development, the displacement of the impulsive mode, the stiffness of the impulsive mode, the displacement of the convective mode, the stiffness of the convective mode and the displacement of the top slab of the foundation, respectively. For calculating the response of the traditional system, the two SDOFs representing the tank were subjected to the ground motions without Metafoundation, which is equivalent to the tank being clamped to a traditional foundation, such as a concrete slab. The maximum base shear during each earthquake was considered as the governing performance measure and recorded for the Metafoundation and traditional foundation layouts with,

$$
\eta=\frac{\sum_{n=1}^{15} \max \left(V(t)_{n}^{\text {meta }}\right)}{\sum_{n=1}^{15} \max \left(V(t)_{n}^{\text {trad }}\right)}
$$

Here, $n$ identifies the earthquake, while the sums simply cumulate the maximum base shear values of each seismic event. Figure 12(a), (b), and (c) shows the results for the FULL, REDUCED, and MINIMAL systems endowed with varying levels of NSE, thereby displaying how the performance measure $\eta$ improves with an increase in NSE value. Moreover, when comparing the various setups without NSE ( $0 \%$ NSE), it becomes clear that the $\eta$ value is significantly lower for the larger systems FULL $\eta=0.79$ and REDUCED $\eta=0.82$ compared to $\eta=0.93$ for the MINIMAL system. This is due to the stiffness of the structure, which is determined by the structural design of the columns, limiting the minimal size of the Metafoundation without NSEs. However, when considering the positive impact that the NSE has on the system performance, it becomes possible to achieve a similar performance with the MINIMAL system with 50\% of the maximal allowable NSE value. Furthermore, it is interesting to observe that the maximal recorded base shear for each earthquake tends to show less 
variation for higher NSE values. This suggests that with greater NSE, the performance of the system becomes more reliable across various seismic records, which is a very desirable property, due to the extreme variability of frequency and amplitude content of earthquakes.

In Subsection 3.2.2 we mentioned that the introduction of the 3rd harmonic may transfer some energy from the first mode to this higher harmonic, and therefore, has the potential to improve the systems performance. However, the investigated systems FULL, REDUCED and MINIMAL do not enter the strongly nonlinear range, and therefore, further calculations are carried out herein. A study of the two established nonlinear parameters $l$ and $\epsilon$ is conducted for $l$ being equal to $1,0.1,0.09,0.05,0.02$, and $0.01 \mathrm{~m}$, and $\epsilon$ being equal to $0.5,0.9$, and 0.99 . These values are applied to the FULL system with $75 \%$ NSE, while the results are reported in Figure 13(a), (b), and (c). Although these values are geometrically very unrealistic for the system under study, other applications with a different scale may reach the nonlinear range, and therefore, may profit from the nonlinear effects. However, Figure 13(a), (b), and (c) depicts the trend of the system when entering the nonlinear regime and shows clearly that an increase in nonlinearity acts detrimental on the performance of the system. More precisely, there is an obvious degradation of the performance value from $\eta=0.532$ for the system with a compression member length of $1 \mathrm{~m}$, to $\eta=0.90$ for a compression member length of $0.01 \mathrm{~m}$. Curiously, the setups with $l=0.05 \mathrm{~m}$ and $l=0.09 \mathrm{~m}$ show a very small improvement over the quasi 420 linear system for the earthquakes 004673ya and 000594ya. For most other events however, the systems perform significantly worse and an advantage cannot be concluded. On the other hand, the trend of the system with a decrease in $\epsilon$ value shows that the system is less likely to go in the nonlinear range even for very small $l$ values, which may be useful for the design of the mechanism. 

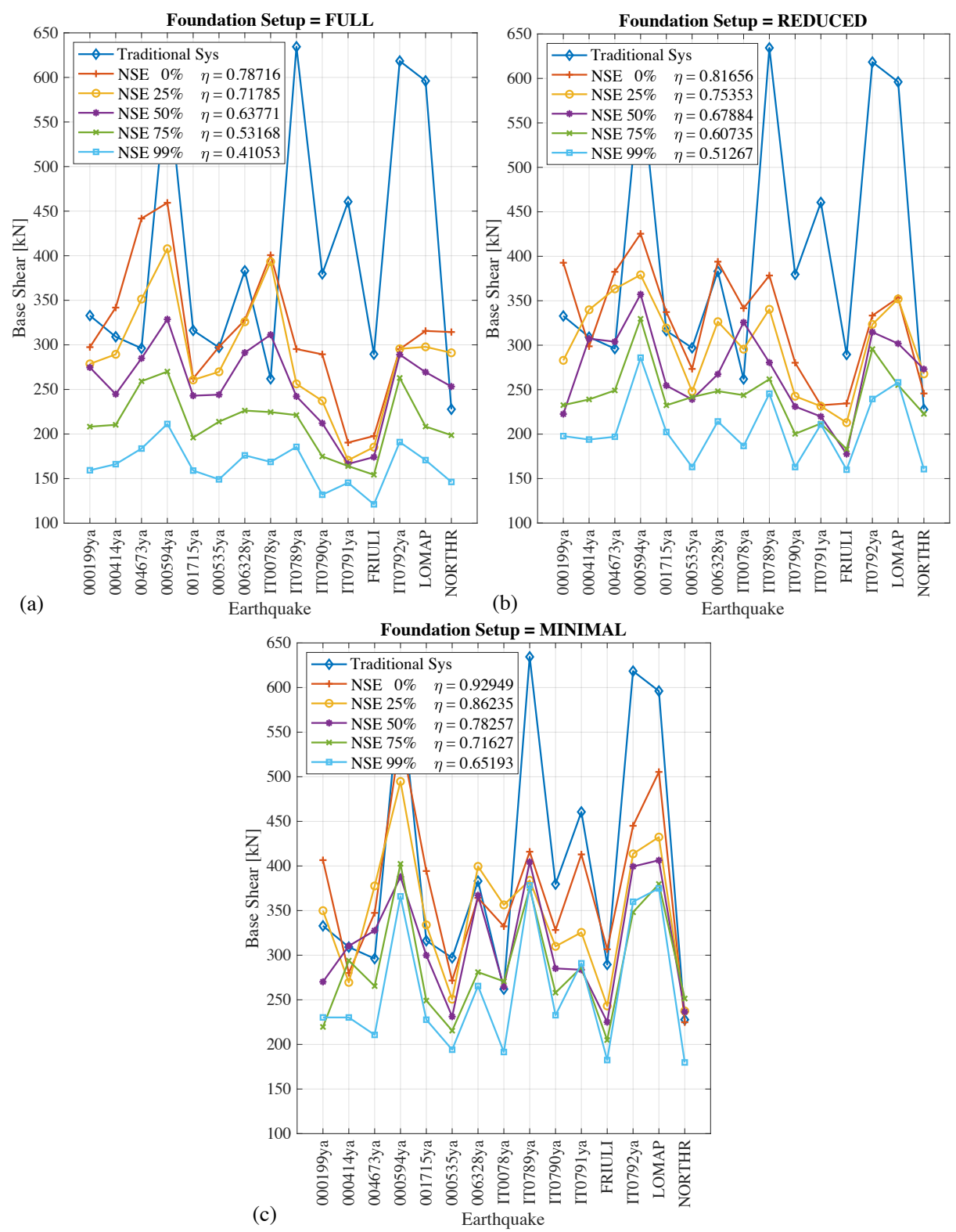

Figure 12: Base shear maxima from time history analyses with $\epsilon=0.95$ and various levels of NSE: (a) FULL system; (b) REDUCED system; (c) MINIMAL system. 


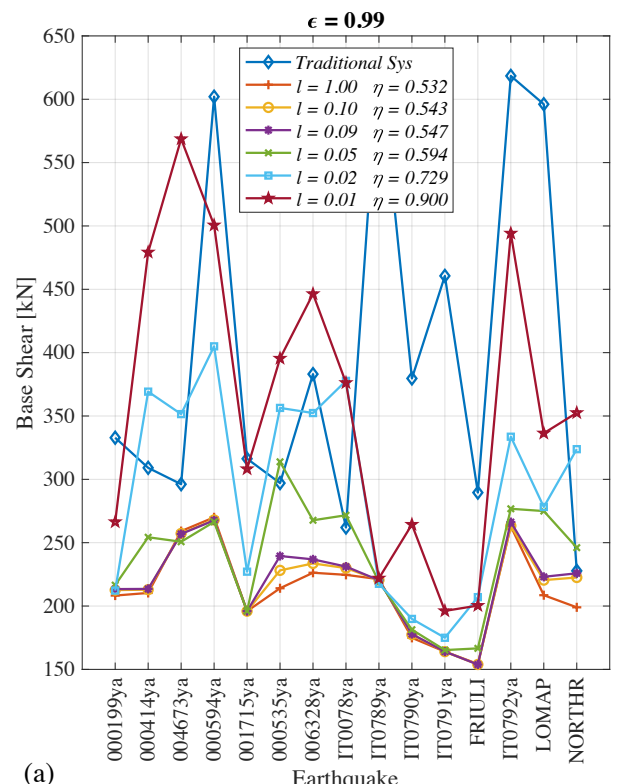

(a)

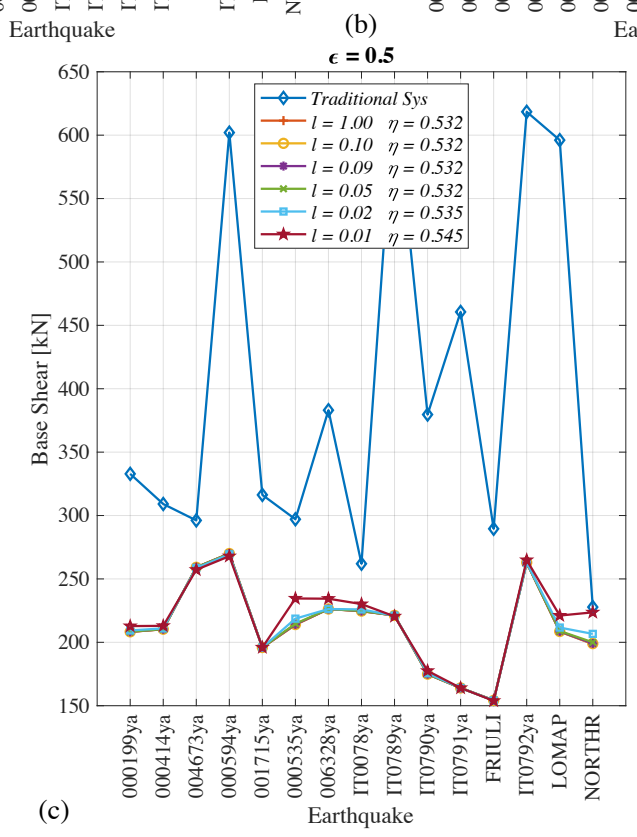

Figure 13: Base shear maxima from time history analyses with various $\epsilon$ and $l$ values for a system with $75 \% \mathrm{NSE}$ : (a) $\epsilon=0.99$; (b) $\epsilon=0.90$; (c) $\epsilon=0.50$. 


\section{Conclusions}

In this work, a new type of NSE, based on a compression member in a stable snap through position, has been developed for the application to seismic metamaterials. The composite system showed enhanced wave attenuation characteristics and was studied on its fully nonlinear behavior via time and frequency domain analyses. Due to the implemented NSE as well as the new type of established resonator chain, the system displayed a widening of the band gap and an amplification of the attenuation capabilities with an increase in NSE. It is further worth noting that a finite system exerted an attenuation zone that stretched into an even lower frequency range than the band gap of the periodic system predicted. The nonlinearity on the other hand, proved to have a detrimental effect on the band gap range, since an increase in activated nonlinearity narrowed the band gap towards its upper bound. Besides the study of the NSE enhanced foundation as a periodic structure, also its application to fuel storage tanks as a seismic protection system was discussed herein. Due to the feedback from the superstructure and the shift of eigenfrequencies caused by the NSE, the tuning of the finite systems necessitated an optimization algorithm. Note that the algorithm proposed herein considered the superstructure as well as the ground motion spectrum and could in principle optimize any number of system parameters. After optimizing the system a set of spectrum compatible ground motions was used to evaluate its performance, which demonstrated that a size reduction to $1 / 3$ of the original size was achievable with $50 \%$ of the physically allowable negative stiffness. Additionally, the NSE enhanced systems showed a more reliable performance across various earthquakes, which is a highly desirable property, due to the strong variability in frequency and amplitude content of seismic records. It is further worth mentioning that the effect of soil structure interaction has been neglected in the present work, by assuming that the foundation is placed on bedrock. However, due to the resonant nature of the foundation, soil structure interaction may provide particularly interesting effects especially for vertical component damping, and therefore, deserves to be 

was designed in a general way and may be applied to other vibration problems, where shear type waves need to be attenuated.

\section{Acknowledgements}

This project has received funding from the European Union's Horizon 2020

[1] M. H. Lu, L. Feng, Y. F. Chen, Phononic crystals and acoustic metamaterials, Materials Today 12 (12) (2009) 34-42. doi:10.1016/S1369-7021(09) 70315-3

[2] X. Zhou, X. Liu, G. Hu, Elastic metamaterials with local resonances: an

[ [5] Z. Cheng, Z. Shi, Composite periodic foundation and its application for ${ }_{480} \quad$ seismic isolation, Earthquake Engineering \& Structural Dynamics 47 (4) 
(2018) 925-944. doi:10.1002/eqe.2999.

URL http://doi.wiley.com/10.1002/eqe.2999

[6] J. Bao, Z. Shi, H. Xiang, Dynamic responses of a structure with periodic foundations, Journal of Engineering Mechanics 138 (7) (2012) 761-769. doi:10.1061/(ASCE) EM.1943-7889.0000383

[7] O. Casablanca, G. Ventura, F. Garescì, B. Azzerboni, B. Chiaia, M. Chiappini, G. Finocchio, Seismic isolation of buildings using composite foundations based on metamaterials, Journal of Applied Physics 123 (17) (may 2018). doi:10.1063/1.5018005.

[8] S. H. Kim, M. P. Das, Seismic waveguide of metamaterials, Modern Physics Letters B 26 (17) (jul 2012). arXiv:1202.1586, doi:10.1142/ S0217984912501059

[9] A. Palermo, S. Krödel, A. Marzani, C. Daraio, Engineered metabarrier as

- shield from seismic surface waves, Scientific Reports 6 (dec 2016). doi: $10.1038 /$ srep39356

[10] J. Huang, Z. Shi, Attenuation zones of periodic pile barriers and its application in vibration reduction for plane waves, Journal of Sound and Vibration 332 (19) (2013) 4423-4439. doi:10.1016/j.jsv.2013.03.028

[11] V. K. Dertimanis, I. A. Antoniadis, E. N. Chatzi, Feasibility Analysis on 500 the Attenuation of Strong Ground Motions Using Finite Periodic Lattices of Mass-in-Mass Barriers, Journal of Engineering Mechanics 142 (9) (2016) 04016060. doi:10.1061/(ASCE) EM.1943-7889.0001120.

ص URL http://ascelibrary.org/doi/10.1061/\{\%\}28ASCE\{\%\}29EM. $1943-7889.0001120$

505 [12] A. H. Schellenberg, A. Sarebanha, M. J. Schoettler, G. Mosqueda, G. Benzoni, A. M. Stephen, PEER Report: "Hybrid Simulation of Seismic Isolation Systems Applied to an APR-1400 Nuclear Power Plant", Tech. rep., Pacific Earthquake Engineering Research Center (2015). 
[13] F. Basone, M. Wenzel, O. Bursi, M. Fossetti, Finite locally resonant

[14] European Standard, Eurocode 3: Design of steel structures (2005).

[15] European Standard, Eurocode 8: Design of Structures for Earthquake Re-

[17] W. Witarto, S. J. Wang, C. Y. Yang, X. Nie, Y. L. Mo, K. C. Chang,

1 Y. Tang, R. Kassawara, Seismic isolation of small modular reactors us-

1 ing metamaterials, AIP Advances 8 (4) (2018) 045307. doi:10.1063/1. 5020161 .

[18] B. S. Ungureanu, Y. Achaoui, S. Enoch, S. Brûlé, S. Guenneau, A. Christos, M. Alessio, Auxetic-like metamaterials as novel earthquake protections, EPJ Applied Metamaterials (jan 2015). doi:10.1051/EPJAM/2016001

[19] D. Mu, H. Shu, L. Zhao, S. An, A Review of Research on Seismic 530

I URL https://onlinelibrary.wiley.com/doi/abs/10.1002/adem. 201901148

[20] Y. Choi, J.-B. Park, S.-J. Lee, N.-C. Park, Y.-P. Park, J.-S. Kim, W.-J.

1 Roh, Seismic analysis of the APR 1400 reactor vessel internals using

${ }_{535}$ the model reduction method, Journal of Nuclear Science and Technology 53 (11) (2016) 1701-1714. doi:10.1080/00223131.2016.1152921. 
URL https://www.tandfonline.com/doi/full/10.1080/00223131. 2016.1152921

[21] T. Fukasawa, S. Okamura, T. Somaki, T. Miyagawa, M. Uchita, T. Yamamoto, T. Watakabe, S. Fujita, Research and development of threedimensional isolation system for sodium-cooled fast reactor: Part 4 - Proposal of optimal combination method for disc spring units and newly friction model for sliding elements, in: American Society of Mechanical Engineers, Pressure Vessels and Piping Division (Publication) PVP,

${ }_{545}$ Vol. 8, American Society of Mechanical Engineers (ASME), 2019. doi: 10.1115/PVP2019-93480.

[22] I. Antoniadis, D. Chronopoulos, V. Spitas, D. Koulocheris, Hyper-damping properties of a stiff and stable linear oscillator with a negative stiffness

1. element, Journal of Sound and Vibration 346 (1) (2015) 37-52. doi:10. $1016 / j \cdot j s v .2015 .02 .028$.

[23] J. H. Oh, Y. E. Kwon, H. J. Lee, Y. Y. Kim, Elastic metamaterials for independent realization of negativity in density and stiffness, Scientific Reports 6 (mar 2016). doi:10.1038/srep23630.

[24] C. Ren, D. Yang, H. Qin, Mechanical performance of multidirectional Buckling-based Negative Stiffness metamaterials: An analytical and numerical study, Materials 11 (7) (jun 2018). doi:10.3390/ma11071078

[25] G. H. Yoon, J. S. Mo, Tailoring a bidirectional negative stiffness (BNS) structure with mechanical diodes for mechanical metamaterial struc-

1 tures, Smart Materials and Structures 26 (5) (apr 2017). doi:10.1088/ 560 1361-665X/aa65bf.

[26] C. Morris, L. Bekker, C. Spadaccini, M. Haberman, C. Seepersad, Tunable 1. Mechanical Metamaterial with Constrained Negative Stiffness for Improved Quasi-Static and Dynamic Energy Dissipation, Advanced Engineering Materials 21 (7) (2019) 1900163. doi:10.1002/adem.201900163. 
URL https://onlinelibrary.wiley.com/doi/abs/10.1002/adem. 201900163

[27] G. Chakraborty, A. K. Mallik, Dynamics of a weakly non-linear periodic chain, International Journal of Non-Linear Mechanics 36 (2) (2001) 375389. doi:10.1016/S0020-7462(00)00024-X.

[28] B. S. Lazarov, J. S. Jensen, Low-frequency band gaps in chains with attached non-linear oscillators, International Journal of Non-Linear Mechanics 42 (10) (2007) 1186-1193. doi:10.1016/j.ijnonlinmec.2007.09.007.

[29] A. Banerjee, R. Das, E. P. Calius, Waves in Structured Mediums or Metamaterials: A Review, Archives of Computational Methods in Engineering (2018) 1-30 doi:10.1007/s11831-018-9268-1.

[30] A. Barka, The 17 August 1999 Izmit earthquake, Science 285 (5435) (1999) 1858-1859. doi:10.1126/science.285.5435.1858.

[31] M. Nakashima, O. Lavan, M. Kurata, Y. Luo, Earthquake engineering research needs in light of lessons learned from the 2011 Tohoku earthquake, Earthquake Engineering and Engineering Vibration 13 (1) (2014) 141-149. doi:10.1007/s11803-014-0244-y.

[32] A. M. Cruz, L. J. Steinberg, A. L. Vetere-Arellano, Emerging issues for natech disaster risk management in Europe, Journal of Risk Research 9 (5) (2006) 483-501. doi:10.1080/13669870600717657.

[33] L. J. Steinberg, H. Sengul, A. M. Cruz, Natech risk and management: An assessment of the state of the art, Natural Hazards 46 (2) (2008) 143-152. doi:10.1007/s11069-007-9205-3.

[34] V. Cozzani, G. Antonioni, G. Landucci, A. Tugnoli, S. Bonvicini, G. Spadoni, Quantitative assessment of domino and NaTech scenarios in complex industrial areas, Journal of Loss Prevention in the Process Industries 28 (2014) 10-22. doi:10.1016/j.jlp.2013.07.009. 
[35] P. K. Malhotra, T. Wenk, M. Wieland, Simple procedure for seismic analysis of liquid-storage tanks, Structural Engineering International: Journal of the International Association for Bridge and Structural Engineering (IABSE) 10 (3) (2000) 197-201. doi:10.2749/101686600780481509.

[36] A. S. Phani, J. Woodhouse, N. A. Fleck, Wave propagation in twodimensional periodic lattices, The Journal of the Acoustical Society of America 119 (4) (2006) 1995-2005. doi:10.1121/1.2179748.

[37] G. Sen Gupta, Natural flexural waves and the normal modes of periodicallysupported beams and plates, Journal of Sound and Vibration 13 (1) (1970) 89-101. doi:10.1016/S0022-460X (70)80082-7.

[38] V. S. Sorokin, J. J. Thomsen, Effects of weak nonlinearity on the dispersion relation and frequency band-gaps of a periodic Bernoulli-Euler beam, Proceedings of the Royal Society A: Mathematical, Physical and Engineering Sciences 472 (2186) (feb 2016). doi:10.1098/rspa.2015.0751.

[39] J. B. Roberts, P. D. Spanos, Random vibration and statistical linearization, Wiley, Chichester ;New York, 1990.

[40] R. W. Clough, J. Penzien, Dynamics of structures, McGraw-Hill, 1975. 